\title{
Definitizability of Normal Operators on Krein Spaces and Their Functional Calculus
}

\author{
Michael Kaltenbäck
}

\begin{abstract}
We discuss a new concept of definitizability of a normal operator on Krein spaces. For this new concept we develop a functional calculus $\phi \mapsto \phi(N)$ which is the proper analogue of $\phi \mapsto \int \phi d E$ in the Hilbert space situation.
\end{abstract}

Mathematics Subject Classification. 47A60, 47B50, 47B15.

Keywords. Krein space, Definitizable operators, Normal operators, Spectral theorem.

\section{Introduction}

A bounded linear operator $N$ on a $\operatorname{Krein}$ space $(\mathcal{K},[.,]$.$) is normal, if N$ commutes with its Krein space adjoint $N^{+}$. If we write a bounded linear $N$ as $A+i B$ with the selfadjoint real part $A:=\operatorname{Re} N:=\frac{N+N^{+}}{2}$ and the selfadjoint imaginary part $B:=\operatorname{Im} N:=\frac{N-N^{+}}{2 i}$, then $N$ is normal if and only if $A B=B A$. In [4] we called a normal $N$ definitizable whenever $A$ and $B$ were both definitizable in the classical sense, i.e. there exist so-called definitizing polynomials $p(z), q(z) \in \mathbb{R}[z] \backslash\{0\}$ such that $[p(A) x, x] \geq 0$ and $[q(B) x, x] \geq 0$ for all $x \in \mathcal{K}$.

For such definitizable operators in [4] we could build a functional calculus in analogy to the functional calculus $\phi \mapsto \int \phi d E$ mapping the $*$-algebra of bounded and measurable functions on $\sigma(N)$ to $B(\mathcal{H})$ in the Hilbert space case. The functional calculus in [4] can also be seen as a generalization of Heinz Langers spectral theorem on definitizable selfadjoint operators on Krein spaces; see $[5,6]$. Unfortunately, there are unsatisfactory phenomenons with this concept of definitizability in [4]. For example, it is not clear, whether for a bijective, normal definitizable $N$ also $N^{-1}$ definitizable.

In the present paper we choose a more general concept of definitizability. We shall say that a normal $N$ on a Krein space $\mathcal{K}$ is definitizable if $[p(A, B) u, u] \geq 0$ for all $u \in \mathcal{K}$ for some, so-called definitizing, $p \in \mathbb{C}[x, y] \backslash\{0\}$

This work was supported by a joint project of the Austrian Science Fund (FWF, I1536N25) and the Russian Foundation for Basic Research (RFBR, 13-01-91002-ANF). 
with real coefficients. Then we study the ideal $\mathcal{I}$ generated by all definitizing polynomials with real coefficients in $\mathbb{C}[x, y]$, and assume that $\mathcal{I}$ is large in the sense that it is zero-dimensional, i.e. $\operatorname{dim} \mathbb{C}[x, y] / \mathcal{I}<\infty$. By the way, if $N$ is definitizable in the sense of [4], then $\mathcal{I}$ is always zero-dimensional.

Using results from algebraic geometry, under the assumption that $\mathcal{I}$ is zero-dimensional, the variety $V(\mathcal{I})=\left\{a \in \mathbb{C}^{2}: f(a)=0\right.$ for all $\left.f \in \mathcal{I}\right\}$ is a finite set. We split this subset of $\mathbb{C}^{2}$ up as

$$
V(\mathcal{I})=\left(V(\mathcal{I}) \cap \mathbb{R}^{2}\right) \dot{U}\left(V(\mathcal{I}) \backslash \mathbb{R}^{2}\right),
$$

and interpret $V_{\mathbb{R}}(\mathcal{I}):=V(\mathcal{I}) \cap \mathbb{R}^{2}$ in the following as a subset of $\mathbb{C}$ by considering the first entry of an element of $\mathbb{R}^{2}$ as the real and the second entry as the imaginary part.

Due to the ascending chain condition the ideal $\mathcal{I}$ is generated by finitely many real definitizing polynomials $p_{1}, \ldots, p_{m}$. With the help of the positive semidefinite scalar products $\left[p_{j}(A, B) .,.\right], j=1, \ldots, m$, and $\sum_{k=1}^{m}\left[p_{k}(A, B) .,.\right]$ we construct Hilbert spaces $\mathcal{H}_{j}, j=1, \ldots, m$, and $\mathcal{H}$ together with bounded and injective $T_{j}: \mathcal{H}_{j} \rightarrow \mathcal{K}$ and $T: \mathcal{H} \rightarrow \mathcal{K}$. We consider the $*$-algebra homomorphisms $\Theta_{j}:\left(T_{j} T_{j}^{+}\right)^{\prime} \rightarrow\left(T_{j}^{+} T_{j}\right)^{\prime}, C \mapsto\left(T_{j} \times T_{j}\right)^{-1}(C)$ and $\Theta$ : $\left(T T^{+}\right)^{\prime} \rightarrow\left(T^{+} T\right)^{\prime}, C \mapsto(T \times T)^{-1}(C)$ as studied in [5],

Here $T_{j} \times T_{j}: \mathcal{H}_{j} \times \mathcal{H}_{j} \rightarrow \mathcal{K} \times \mathcal{K}$ maps the pair $(x ; y)$ to the pair $\left(T_{j} x ; T_{j} y\right)$ and $T \times T: \mathcal{H} \times \mathcal{H} \rightarrow \mathcal{K} \times \mathcal{K}$ maps $(x ; y)$ to $(T x ; T y)$. By $\left(T_{j} T_{j}^{+}\right)^{\prime},\left(T T^{+}\right)^{\prime} \subseteq$ $B(\mathcal{K})$ and $\left(T_{j}^{+} T_{j}\right)^{\prime} \subseteq B\left(\mathcal{H}_{j}\right),\left(T^{+} T\right)^{\prime} \subseteq B(\mathcal{H})$ we denote the commutant of the respective operators.

The proper family $\mathcal{F}_{N}$ of functions suitable for the aimed functional calculus are functions defined on

$$
\left(\sigma(\Theta(N)) \cup V_{\mathbb{R}}(\mathcal{I})\right) \dot{\cup}\left(V(\mathcal{I}) \backslash \mathbb{R}^{2}\right) .
$$

Moreover, the functions $\phi \in \mathcal{F}_{N}$ assume values in $\mathbb{C}$ on $\sigma(\Theta(N)) \backslash V_{\mathbb{R}}(\mathcal{I})$ and values in certain finite dimensional $*$-algebras $\mathcal{A}(z)$ at $z \in V_{\mathbb{R}}(\mathcal{I})$ and $\mathcal{B}((\xi, \eta))$ at $(\xi, \eta) \in V(\mathcal{I}) \backslash \mathbb{R}^{2}$. On $\sigma(\Theta(N)) \backslash V_{\mathbb{R}}(\mathcal{I})$ we assume $\phi$ to be bounded and measurable. Finally, $\phi \in \mathcal{F}_{N}$ satisfies a growth regularity condition at all $w$ points from $V_{\mathbb{R}}(\mathcal{I})$ which are not isolated in $\sigma(\Theta(N)) \cup V_{\mathbb{R}}(\mathcal{I})$. Vaguely speaking, this growth regularity condition means that around $w$ the function $\phi$ admits an approximation by a Taylor polynomial, which is determined by $\phi(w) \in \mathcal{A}(w)$. Any polynomial $s \in \mathbb{C}[x, y]$ can be seen as a function $s_{N} \in \mathcal{F}_{N}$ in a natural way.

For each $\phi \in \mathcal{F}_{N}$ we will see that there exist $p \in \mathbb{C}[x, y]$ and bounded, measurable $f_{1}, \ldots, f_{m}: \sigma(\Theta(N)) \cup V_{\mathbb{R}}(\mathcal{I}) \rightarrow \mathbb{C}$ with $f_{j}(z)=0$ for $z \in V_{\mathbb{R}}(\mathcal{I})$ such that

$$
\phi(z)=p_{N}(z)+\sum_{j} f_{j}(z)\left(p_{j}\right)_{N}(z)
$$

for all $z \in \sigma(\Theta(N)) \cup V_{\mathbb{R}}(\mathcal{I})$, and that $\phi((\xi, \eta))=p_{N}((\xi, \eta))$ for all $(\xi, \eta) \in$ $V(\mathcal{I}) \backslash \mathbb{R}^{2}$. Defining $(E$ denotes the spectral measure of $\Theta(N)$ )

$$
\phi(N):=p(A, B)+\sum_{k=1}^{m} T_{k}\left(\int_{\sigma\left(\Theta_{k}(N)\right)} f_{k} d E_{k}\right) T_{k}^{+},
$$


we show that this operator does not depend on the actual decomposition (1.1) and that $\phi \mapsto \phi(N)$ is indeed a $*$-homomorphism satisfying $\phi(N)=s(A, B)$ for $\phi=s_{N}$.

\section{Multiple Embeddings}

In the present section $(\mathcal{K},[.,]$.$) will be a Krein space and (\mathcal{H},(.,)),.\left(\mathcal{H}_{j},(.,).\right)$, $j=1, \ldots, m$, will denote Hilbert spaces. Moreover, let $T: \mathcal{H} \rightarrow \mathcal{K}, T_{j}: \mathcal{H}_{j} \rightarrow$ $\mathcal{K}$ and $R_{j}: \mathcal{H}_{j} \rightarrow \mathcal{H}$ bounded, linear and injective mappings such that $T R_{j}=T_{j}$. By $T^{+}: \mathcal{K} \rightarrow \mathcal{H}$ and $T_{j}^{+}: \mathcal{K} \rightarrow \mathcal{H}_{j}$ we denote the respective Krein space adjoints.

If $D$ is an operator on a Krein space, then we shall denote by $D^{\prime}$ the commutant of $D$, i.e. the algebra of all operators commuting with $D$. For a selfadjoint $D$ this commutant is a $*$-algebra with respect to forming adjoint operators.

For $j=1, \ldots, m$ we shall denote by $\Theta_{j}:\left(T_{j} T_{j}^{+}\right)^{\prime}(\subseteq B(\mathcal{K})) \rightarrow\left(T_{j}^{+} T_{j}\right)^{\prime}$ $\left(\subseteq B\left(\mathcal{H}_{j}\right)\right)$, and by $\Theta:\left(T T^{+}\right)^{\prime}(\subseteq B(\mathcal{K})) \rightarrow\left(T^{+} T\right)^{\prime}(\subseteq B(\mathcal{H}))$ the $*$-algebra homomorphisms mapping the identity operator to the identity operator as in Theorem 5.8 from [5] corresponding to the mappings $T_{j}$ and $T$ :

$$
\begin{aligned}
\Theta_{j}\left(C_{j}\right) & =\left(T_{j} \times T_{j}\right)^{-1}\left(C_{j}\right)=T_{j}^{-1} C_{j} T_{j}, \quad C_{j} \in\left(T_{j} T_{j}^{+}\right)^{\prime}, \\
\Theta(C) & =(T \times T)^{-1}(C)=T^{-1} C T, \quad C \in\left(T T^{+}\right)^{\prime} .
\end{aligned}
$$

We can apply Theorem 5.8 in [5] also to the bounded linear, injective $R_{j}$ : $\mathcal{H}_{j} \rightarrow \mathcal{H}$, and denote the corresponding $*$-algebra homomorphisms by $\Gamma_{j}$ : $\left(R_{j} R_{j}^{*}\right)^{\prime}(\subseteq B(\mathcal{H})) \rightarrow\left(R_{j}^{*} R_{j}\right)^{\prime}\left(\subseteq B\left(\mathcal{H}_{j}\right)\right):$

$$
\Gamma_{j}(D)=\left(R_{j} \times R_{j}\right)^{-1}(D)=R_{j}^{-1} D R_{j}, \quad D \in\left(R_{j} R_{j}^{*}\right)^{\prime} .
$$

For the following note that due to $\left(\operatorname{ran} T^{+}\right)^{[\perp]}=\operatorname{ker} T=\{0\}$ the range of $T^{+}$is dense in $\mathcal{H}$.

Lemma 2.1. For $j=1, \ldots, m$ we have $\Theta\left(\left(T_{j} T_{j}^{+}\right)^{\prime} \cap\left(T T^{+}\right)^{\prime}\right) \subseteq\left(R_{j} R_{j}^{*}\right)^{\prime} \cap$ $\left(T^{+} T\right)^{\prime}$, where in fact

$$
\Theta(C) R_{j} R_{j}^{*}=R_{j} \Theta_{j}(C) R_{j}^{*}=R_{j} R_{j}^{*} \Theta(C), \quad C \in\left(T_{j} T_{j}^{+}\right)^{\prime} \cap\left(T T^{+}\right)^{\prime} .
$$

Moreover,

$$
\Theta_{j}(C)=\left(\Gamma_{j} \circ \Theta\right)(C), \quad C \in\left(T_{j} T_{j}^{+}\right)^{\prime} \cap\left(T T^{+}\right)^{\prime} .
$$

Proof. According to Theorem 5.8 in [5] we have $\Theta_{j}(C) T_{j}^{+}=T_{j}^{+} C$ and $\Theta(C) T^{+}=T^{+} C$ for $C \in\left(T_{j} T_{j}^{+}\right)^{\prime} \cap\left(T T^{+}\right)^{\prime}$. Therefore,

$$
\begin{aligned}
T\left(R_{j} \Theta_{j}(C) R_{j}^{*}\right) T^{+} & =T_{j} \Theta_{j}(C) T_{j}^{+}=T_{j} T_{j}^{+} C \\
& =T R_{j} R_{j}^{*} T^{+} C=T\left(R_{j} R_{j}^{*} \Theta(C)\right) T^{+} .
\end{aligned}
$$

Because of ker $T=\{0\}$ and by the density of $\operatorname{ran} T^{+}$we have $R_{j} \Theta_{j}(C) R_{j}^{*}=$ $R_{j} R_{j}^{*} \Theta(C)$. Applying this equation to $C^{+}$and taking adjoints yields $R_{j} \Theta_{j}(C)$ $R_{j}^{*}=\Theta(C) R_{j} R_{j}^{*}$. In particular, $\Theta(C) \in\left(R_{j} R_{j}^{*}\right)^{\prime}$. Therefore, we can apply $\Gamma_{j}$ to $\Theta(C)$ and get 


$$
\left(\Gamma_{j} \circ \Theta\right)(C)=R_{j}^{-1} T^{-1} C T R_{j}=T_{j}^{-1} C T_{j}=\Theta_{j}(C) .
$$

For the following Corollary 2.3 note that by (2.3) and by the fact that $\Gamma_{j}$ is a $*$-algebra homomorphism mapping the identity operator to the identity operator, for $j=1, \ldots, m$ we have

$$
\sigma(\Theta(C)) \supseteq \sigma\left(\Theta_{j}(C)\right) \text { for all } C \in\left(T_{j} T_{j}^{+}\right)^{\prime} \cap\left(T T^{+}\right)^{\prime} .
$$

Remark 2.2. We would also like to make some clarifications regarding to the integrals over spectral measures. If $E$ is a spectral measure on a Hilbert space $\mathcal{H}$ defined on the Borel subsets of $\mathbb{C}$ such that $E(\mathbb{C} \backslash K)=0$ for some measurable subset $K \subseteq \mathbb{C}$ and if $h: \operatorname{dom} h \rightarrow \mathbb{C}$ is a Borel measurable function with a Borel measurable $\operatorname{dom} h \subseteq \mathbb{C}$ such that $K \subseteq \operatorname{dom} h$ and such that $h$ is bounded on $K$, then $(x ; y) \mapsto \int_{\operatorname{dom} h} h d(E x, y)$ is bounded sesquilinear form on $\mathcal{H}$. Hence,

$$
\int h d E:=\int_{\operatorname{dom} h} h d E
$$

is a well defined bounded operator on $\mathcal{H}$. Clearly, $\int h d E=\int_{K} h d E$. If $E$ is the spectral measure for a bounded normal operator $L$ on $\mathcal{H}$, then this considerations apply for each measurable superset $K$ of $\sigma(L)$.

Corollary 2.3. For $j \in\{1, \ldots, m\}$ let $N \in B(\mathcal{K})$ be normal, i.e. $N N^{+}=$ $N^{+} N$, such that $N \in\left(T_{j} T_{j}^{+}\right)^{\prime} \cap\left(T T^{+}\right)^{\prime}$. Then $\Theta(N)$ is a normal operator on the Hilbert space $\mathcal{H}$, and $\Theta_{j}(N)$ is a normal operator on the Hilbert space $\mathcal{H}_{j}$. Denoting by $E\left(E_{j}\right)$ the spectral measure of $\Theta(N)\left(\Theta_{j}(N)\right)$, we have $E(\Delta) \in\left(R_{j} R_{j}^{*}\right)^{\prime} \cap\left(T^{+} T\right)^{\prime}$ and

$$
\Gamma_{j}(E(\Delta))=E_{j}(\Delta),
$$

for all Borel subsets $\Delta$ of $\mathbb{C}$, and $E_{j}(\Delta) \in\left(R_{j}^{*} R_{j}\right)^{\prime} \cap\left(T_{j}^{+} T_{j}\right)^{\prime}$.

Moreover, $\int h d E \in\left(R_{j} R_{j}^{*}\right)^{\prime} \cap\left(T^{+} T\right)^{\prime}$ and

$$
\Gamma_{j}\left(\int h d E\right)=\int h d E_{j}
$$

for any bounded and measurable $h: \sigma(\Theta(N)) \rightarrow \mathbb{C}$, and $\int h d E_{j} \in\left(R_{j}^{*} R_{j}\right)^{\prime} \cap$ $\left(T_{j}^{+} T_{j}\right)^{\prime}$.

Proof. The normality of $\Theta(N)$ and $\Theta_{j}(N)$ is clear, since $\Theta$ and $\Theta_{j}$ are *homomorphisms. From Lemma 2.1 we know that $\Theta(N) \in\left(R_{j} R_{j}^{*}\right)^{\prime} \cap\left(T^{+} T\right)^{\prime}$. According to the well known properties of $\Theta(N)$ 's spectral measure we obtain $E(\Delta) \in\left(R_{j} R_{j}^{*}\right)^{\prime} \cap\left(T^{+} T\right)^{\prime}$ and, in turn, $\int h d E \in\left(R_{j} R_{j}^{*}\right)^{\prime} \cap\left(T^{+} T\right)^{\prime}$. In particular, $\Gamma_{j}$ can be applied to $E(\Delta)$ and $\int h d E$. Similarly, $\Theta_{j}(N) \in\left(T_{j}^{+} T_{j}\right)^{\prime}$ implies $E_{j}(\Delta), \int h d E_{j} \in\left(T_{j}^{+} T_{j}\right)^{\prime}$ for a bounded and measurable $h$.

Recall from Theorem 5.8 in [5] that $\Gamma_{j}(D) R_{j}^{*} x=R_{j}^{*} D$ for $D \in\left(R_{j} R_{j}^{*}\right)^{\prime}$. Hence, for $x \in \mathcal{H}$ and $y \in \mathcal{H}_{j}$ we have

$$
\left(\Gamma_{j}(E(\Delta)) R_{j}^{*} x, y\right)=\left(R_{j}^{*} E(\Delta) x, y\right)=\left(E(\Delta) x, R_{j} y\right)
$$


and, in turn,

$$
\begin{aligned}
\int_{\mathbb{C}} s(z, \bar{z}) d\left(\Gamma_{j}(E) R_{j}^{*} x, y\right) & =\int_{\mathbb{C}} s(z, \bar{z}) d\left(E x, R_{j} y\right) \\
& =\left(s\left(\Theta(N), \Theta(N)^{*}\right) x, R_{j} y\right) \\
& =\left(R_{j}^{*} s\left(\Theta(N), \Theta(N)^{*}\right) x, y\right) \\
& =\left(\Gamma_{j}\left(s\left(\Theta(N), \Theta(N)^{*}\right)\right) R_{j}^{*} x, y\right)
\end{aligned}
$$

for any $s \in \mathbb{C}[z, w]$. By (2.3) and the fact, that $\Gamma_{j}$ is a $*$-homomorphism, we have $\Gamma_{j}\left(s\left(\Theta(N), \Theta(N)^{*}\right)\right)=s\left(\Theta_{j}(N), \Theta_{j}(N)^{*}\right)$. Consequently,

$$
\int_{\mathbb{C}} s(z, \bar{z}) d\left(\Gamma_{j}(E) R_{j}^{*} x, y\right)=\int_{\mathbb{C}} s(z, \bar{z}) d\left(E_{j} R_{j}^{*} x, y\right) .
$$

Since $E(\mathbb{C} \backslash K)=0$ and $E_{j}(\mathbb{C} \backslash K)=0$ for a certain compact $K \subseteq \mathbb{C}$ and since the set of all $s(z, \bar{z}), s \in \mathbb{C}[z, w]$, is densely contained in $C(K)$, we obtain from the uniqueness assertion in the Riesz Representation Theorem

$$
\left(\Gamma_{j}(E(\Delta)) R_{j}^{*} x, y\right)=\left(E_{j}(\Delta) R_{j}^{*} x, y\right) \quad \text { for all } \quad x \in \mathcal{H}, y \in \mathcal{H}_{j},
$$

for all Borel subsets $\Delta$ of $\mathbb{C}$. Due to the density of $\operatorname{ran} R_{j}^{*}$ in $\mathcal{H}_{j}$ we even have $\left(\Gamma_{j}(E(\Delta)) v, y\right)=\left(E_{j}(\Delta) v, y\right)$ for all $y, v \in \mathcal{H}_{j}$, and in turn $\Gamma_{j}(E(\Delta))=$ $E_{j}(\Delta)$. Since $\Gamma_{j}$ maps into $\left(R_{j}^{*} R_{j}\right)^{\prime}$, we have $E_{j}(\Delta) \in\left(R_{j}^{*} R_{j}\right)^{\prime}$. This yields $\int h d E_{j} \in\left(R_{j}^{*} R_{j}\right)^{\prime}$ for any bounded and measurable $h$.

If $h: \sigma(\Theta(N)) \rightarrow \mathbb{C}$ is bounded and measurable, then by (2.4) also its restriction to $\sigma\left(\Theta_{j}(N)\right)=\sigma\left(\left(\Gamma_{j} \circ \Theta\right)(N)\right)$ is bounded and measurable. Due to $E_{j}(\Delta) R_{j}^{*}=\Gamma_{j}(E(\Delta)) R_{j}^{*}=R_{j}^{*} E(\Delta)$, for $x \in \mathcal{H}$ and $y \in \mathcal{H}_{j}$ we have

$$
\begin{aligned}
\left(\Gamma_{j}\left(\int h d E\right) R_{j}^{*} x, y\right) & =\left(R_{j}^{*}\left(\int h d E\right) x, y\right) \\
& =\left(\left(\int h d E\right) x, R_{j} y\right) \\
& =\int h d\left(E x, R_{j} y\right)=\int h d\left(E_{j} R_{j}^{*} x, y\right) \\
& =\left(\left(\int h d E_{j}\right) R_{j}^{*} x, y\right) .
\end{aligned}
$$

The density of $\operatorname{ran} R_{j}^{*}$ yields $\Gamma_{j}\left(\int h d E\right)=\int h d E_{j}$.

Recall from Lemma 5.11 in [5] the mappings $(j=1, \ldots, m)$

$$
\begin{gathered}
\Xi_{j}: B\left(\mathcal{H}_{j}\right) \rightarrow B(\mathcal{K}), \quad \Xi_{j}\left(D_{j}\right)=T_{j} D_{j} T_{j}^{+}, \\
\Xi: B(\mathcal{H}) \rightarrow B(\mathcal{K}), \quad \Xi(D)=T D T^{+} .
\end{gathered}
$$

By $(j=1, \ldots, m)$

$$
\Lambda_{j}: B\left(\mathcal{H}_{j}\right) \rightarrow B(\mathcal{H}), \quad \Lambda_{j}\left(D_{j}\right)=R_{j} D_{j} R_{j}^{*},
$$

we shall denote the corresponding mappings outgoing from the mappings $R_{j}: \mathcal{H}_{j} \rightarrow \mathcal{H}$. Due to $T_{j}=T R_{j}$ we have $\Xi_{j}=\Xi \circ \Lambda_{j}$. 
According to Lemma 5.11 in $[5],\left(\Lambda_{j} \circ \Gamma_{j}\right)(D)=D R_{j} R_{j}^{*}$ for $D \in\left(R_{j} R_{j}^{*}\right)^{\prime}$. Hence, using the notation from Corollary 2.3,

$$
\Xi_{j}\left(\int h d E_{j}\right)=\Xi\left(\left(\Lambda_{j} \circ \Gamma_{j}\right)\left(\int h d E\right)\right)=\Xi\left(R_{j} R_{j}^{*} \int h d E\right) .
$$

Lemma 2.4. Assume that for $j \in\{1, \ldots, m\}$ the operator $T_{j} T_{j}^{+}$commutes with $T T^{+}$on $\mathcal{K}$. Then the operators $R_{j} R_{j}^{*}, T^{+} T$ commute on $\mathcal{H}$ and $R_{j}^{*} R_{j}$, $T_{j}^{+} T_{j}$ commute on $\mathcal{H}_{j}$. Moreover,

$$
\Theta\left(T_{j} T_{j}^{+}\right)=R_{j} R_{j}^{*} T^{+} T=T^{+} T R_{j} R_{j}^{*} .
$$

Proof. If $T_{j} T_{j}^{+}$and $T T^{+}$commute on $\mathcal{K}$, then

$$
T\left(T^{+} T R_{j} R_{j}^{*}\right) T^{+}=T T^{+} T_{j} T_{j}^{+}=T_{j} T_{j}^{+} T T^{+}=T\left(R_{j} R_{j}^{*} T^{+} T\right) T^{+} .
$$

Employing $T$ 's injectivity and the density of $\operatorname{ran} T^{+}$, we see that $R_{j} R_{j}^{*}$ and $T^{+} T$ commute. From this we derive

$$
T_{j}^{+} T_{j} R_{j}^{*} R_{j}=R_{j}^{*}\left(T^{+} T R_{j} R_{j}^{*}\right) R_{j}=R_{j}^{*}\left(R_{j} R_{j}^{*} T^{+} T\right) R_{j}=R_{j}^{*} R_{j} T_{j}^{+} T_{j} .
$$

(2.7) follows from

$$
{ }^{-1} T_{j} T_{j}^{+} T=T^{-1} T R_{j} R_{j}^{*} T^{+} T=R_{j} R_{j}^{*} T^{+} T .
$$

\section{Definitizability}

In [4] we said that a normal $N \in B(\mathcal{K})$ is definitizable, if its real part $A:=$ $\frac{N+N^{+}}{2}$ and its imaginary part $B:=\frac{N-N^{*}}{2 i}$ are definitizable in the sense that there exist polynomials $p, q \in \mathbb{R}[z] \backslash\{0\}$ such that $[p(A) v, v] \geq 0$ and $[q(B) v, v] \geq 0$ for all $v \in \mathcal{K}$. In the present note we will relax this condition.

Definition 3.1. For a normal $N \in B(\mathcal{K})$ we call $p \in \mathbb{C}[x, y] \backslash\{0\}$ a definitizing polynomial for $N$, if

$$
[p(A, B) v, v] \geq 0 \text { for all } v \in \mathcal{K} .
$$

where $A=\frac{N+N^{+}}{2}$ and $B=\frac{N-N^{+}}{2 i}$. If such a definitizing $p \in \mathbb{C}[x, y] \backslash\{0\}$ exists, then we call $N$ definitizable normal.

Clearly, we could also write $p$ as a polynomial of the variables $N$ and $N^{+}$. Because of $A=A^{+}$and $B=B^{+}$, writing $p$ as a polynomial of the variables $A$ and $B$, has some notational advantages.

Remark 3.2. According to (3.1) the operator $p(A, B) \in B(\mathcal{K})$ must be selfadjoint; i.e. $p(A, B)^{+}=p^{\#}(A, B)$, where $p^{\#}(x, y)=\overline{p(\bar{x}, \bar{y})}$. Hence, $q:=\frac{p+p^{\#}}{2}$ is real, i.e. $q \in \mathbb{R}[x, y] \backslash\{0\}$, and satisfies $q(A, B)=p(A, B)$. Thus, we can assume that a definitizing polynomial is real.

In the present section we assume that $p_{j}(x, y) \in \mathbb{R}[x, y] \backslash\{0\}, j=1, \ldots, m$, are real, definitizing polynomial for $N$. 
Proposition 3.3. With the above assumptions and notation there exist Hilbert spaces $(\mathcal{H},(.,)),.\left(\mathcal{H}_{j},(.,).\right), j=1, \ldots, m$, and bounded linear and injective operators $T: \mathcal{H} \rightarrow \mathcal{K}, T_{j}: \mathcal{H}_{j} \rightarrow \mathcal{K}$, such that

$$
T_{j} T_{j}^{+}=p_{j}(A, B), \quad \text { and } \quad T T^{+}=\sum_{k=1}^{m} T_{k} T_{k}^{+}=\sum_{k=1}^{m} p_{k}(A, B) .
$$

Proof. Let $\left(\mathcal{H}_{j},(.,).\right)$ be the Hilbert space completion of $\mathcal{K} / \operatorname{ker} p_{j}(A, B)$ with respect to $\left[p_{j}(A, B) .,.\right]$ and let $T_{j}: \mathcal{H}_{j} \rightarrow \mathcal{K}$ be the adjoint of the factor mapping $x \mapsto x+\operatorname{ker} p_{j}(A, B)$ of $\mathcal{K}$ into $\mathcal{H}_{j}$. Since $T_{j}^{+}$has dense range, $T_{j}$ must be injective. Similarly, let $(\mathcal{H},(.,)$.$) be the Hilbert space completion$ of $\mathcal{K} /\left(\operatorname{ker} \sum_{k=1}^{m} p_{k}(A, B)\right)$ with respect to $\left[\left(\sum_{k=1}^{m} p_{k}(A, B)\right) .,.\right]$ and let $T$ : $\mathcal{H} \rightarrow \mathcal{K}$ be the injective adjoint of the factor mapping of $\mathcal{K}$ into $\mathcal{H}$.

Finally, (3.2) follows from $\left[T T^{+} x, y\right]=\left(T^{+} x, T^{+} y\right)=(x, y)=$ $\left[\left(\sum_{k=1}^{m} p_{k}(A, B)\right) x, y\right]$ and $\left[T_{j} T_{j}^{+} x, y\right]=\left(T_{j}^{+} x, T_{j}^{+} y\right)=(x, y)=\left[p_{j}(A, B) x, y\right]$ for all $x, y \in \mathcal{K}$.

Since for $x \in \mathcal{K}$ and $j \in\{1, \ldots, m\}$ we have

$$
\left(T^{+} x, T^{+} x\right)=\left[T T^{+} x, x\right]=\sum_{k=1}^{m}\left[T_{k} T_{k}^{+} x, x\right]=\sum_{k=1}^{m}\left(T_{k}^{+} x, T_{k}^{+} x\right) \geq\left(T_{j}^{+} x, T_{j}^{+} x\right),
$$

one easily concludes that $T^{+} x \mapsto T_{j}^{+} x$ constitutes a well-defined, contractive linear mapping from $\operatorname{ran} T^{+}$onto $\operatorname{ran} T_{j}^{+}$. By $\left(\operatorname{ran} T^{+}\right)^{\perp}=\operatorname{ker} T=\{0\}$ and $\left(\operatorname{ran} T_{j}^{+}\right)^{\perp}=\operatorname{ker} T_{j}=\{0\}$ these ranges are dense in the Hilbert spaces $\mathcal{H}$ and $\mathcal{H}_{j}$. Hence, there is a unique bounded linear continuation of $T^{+} x \mapsto T_{j}^{+} x$ to $\mathcal{H}$, which has dense range in $\mathcal{H}_{j}$.

Denoting by $R_{j}$ the adjoint mapping of this continuation, we clearly have $T_{j}=T R_{j}$ and $\operatorname{ker} R_{j} \subseteq \operatorname{ker} T_{j}=\{0\}$. From (3.2) we conclude

$$
T\left(I_{\mathcal{H}}\right) T^{+}=T T^{+}=\sum_{k=1}^{m} T R_{k} R_{k}^{+} T^{+}=T\left(\sum_{k=1}^{m} R_{k} R_{k}^{+}\right) T^{+} .
$$

$\operatorname{ker} T=\{0\}$ and the density of $\operatorname{ran} T^{+}$yield $\sum_{k=1}^{m} R_{k} R_{k}^{*}=I_{\mathcal{H}}$.

Lemma 3.4. With the above notations and assumptions for $j=1, \ldots, m$ there exist injective contractions $R_{j}: \mathcal{H}_{j} \rightarrow \mathcal{H}$ such that $T_{j}=T R_{j}$ and $\sum_{k=1}^{m} R_{k} R_{k}^{*}=I_{\mathcal{H}}$. Moreover, we have

$$
\left\{N, N^{+}\right\}^{\prime}=\{A, B\}^{\prime} \subseteq \bigcap_{k=1, \ldots, m}\left(T_{k} T_{k}^{+}\right)^{\prime} \subseteq\left(T T^{+}\right)^{\prime}
$$

for all $j \in\{1, \ldots, m\}$. Finally,

$$
\begin{aligned}
p_{j}(\Theta(A), \Theta(B)) & =R_{j} R_{j}^{*}\left(\sum_{k=1}^{m} p_{k}(\Theta(A), \Theta(B))\right) \\
& =\left(\sum_{k=1}^{m} p_{k}(\Theta(A), \Theta(B))\right) R_{j} R_{j}^{*},
\end{aligned}
$$


and for any $u \in \mathbb{C}[x, y]$

$$
\begin{aligned}
p_{j}(A, B) u(A, B) & =\Xi_{j}\left(u\left(\Theta_{j}(A), \Theta_{j}(B)\right)\right) \\
& =\Xi\left(R_{j} R_{j}^{*} u(\Theta(A), \Theta(B))\right),
\end{aligned}
$$

where $\Theta:\left(T T^{+}\right)^{\prime}(\subseteq B(\mathcal{K})) \rightarrow\left(T^{+} T\right)^{\prime}(\subseteq B(\mathcal{H}))$ is as in (2.1) and $\Xi$ : $B(\mathcal{H}) \rightarrow B(\mathcal{K})$ as in $(2.5)$.

Proof. The first part was shown above, and (3.3) is clear from Proposition 3.3.

From (2.7)-Lemma 2.4 can be applied since by (3.2) the operators $T_{j} T_{j}^{+}$all commute with $T T^{+}$_and Theorem 5.8 in [5] we get

$$
\begin{aligned}
p_{j}(\Theta(A), \Theta(B)) & =\Theta\left(p_{j}(A, B)\right)=\Theta\left(T_{j} T_{j}^{+}\right)=R_{j} R_{j}^{*} T^{+} T=R_{j} R_{j}^{*} \Theta\left(T T^{+}\right) \\
& =R_{j} R_{j}^{*} \Theta\left(\sum_{k=1}^{m} p_{k}(A, B)\right)=R_{j} R_{j}^{*}\left(\sum_{k=1}^{m} p_{k}(\Theta(A), \Theta(B))\right),
\end{aligned}
$$

where $R_{j} R_{j}^{*}$ commutes with $T^{+} T=\sum_{k=1}^{m} p_{k}(\Theta(A), \Theta(B))$ by Lemma 2.4. Finally, (3.5) follows from (see Lemma 5.11 in [5])

$$
\begin{aligned}
p_{j}(A, B) u(A, B) & =\Xi_{j}\left(\Theta_{j}(u(A, B))\right)=\left(\Xi \circ \Lambda_{j} \circ \Gamma_{j}\right)(\Theta(u(A, B))) \\
& =\Xi\left(R_{j} R_{j}^{*} u(\Theta(A), \Theta(B))\right) .
\end{aligned}
$$

By (3.3) we can apply Corollary 2.3 in the present situation. In particular, $\Theta(N)$ is a normal operator on the Hilbert space $\mathcal{H}$. Property (3.1) for $p=p_{j}, j=1, \ldots, m$, imply certain spectral properties of $\Theta(N)$.

Lemma 3.5. With the above assumptions and notation for $j \in\{1, \ldots, m\}$ we have

$$
\left\{z \in \mathbb{C}:\left|p_{j}(\operatorname{Re} z, \operatorname{Im} z)\right|>\left\|R_{j} R_{j}^{*}\right\| \cdot\left|\sum_{k=1}^{m} p_{k}(\operatorname{Re} z, \operatorname{Im} z)\right|\right\} \subseteq \rho(\Theta(N)) .
$$

In particular, the zeros of $z \mapsto \sum_{k=1}^{m} p_{k}(\operatorname{Re} z, \operatorname{Im} z)$ in $\mathbb{C}$ are contained in $\rho(\Theta(N)) \cup\left\{z \in \mathbb{C}: p_{j}(\operatorname{Re} z, \operatorname{Im} z)=0\right.$ for all $\left.j=1, \ldots, m\right\}$.

Proof. Let $n \in \mathbb{N}$ and set

$$
\Delta_{n}:=\left\{z \in \mathbb{C}:\left|p_{j}(\operatorname{Re} z, \operatorname{Im} z)\right|^{2}>\frac{1}{n}+\left\|R_{j} R_{j}^{*}\right\|^{2} \cdot\left|\sum_{k=1}^{m} p_{k}(\operatorname{Re} z, \operatorname{Im} z)\right|^{2}\right\} .
$$

For $x \in E\left(\Delta_{n}\right)(\mathcal{H})$, where $E$ denotes $\Theta(N)$ 's spectral measure, we then have

$$
\begin{aligned}
\left\|p_{j}(\Theta(A), \Theta(B)) x\right\|^{2} & =\int_{\Delta_{n}}\left|p_{j}(\operatorname{Re} \zeta, \operatorname{Im} \zeta)\right|^{2} d(E(\zeta) x, x) \\
& \geq \int_{\Delta_{n}} \frac{1}{n} d(E(\zeta) x, x)+\left\|R_{j} R_{j}^{*}\right\|^{2} \int_{\Delta_{n}} \\
& \left|\sum_{k=1}^{m} p_{k}(\operatorname{Re} \zeta, \operatorname{Im} \zeta)\right|^{2} d(E(\zeta) x, x) \\
& \geq \frac{1}{n}\|x\|^{2}+\left\|R_{j} R_{j}^{*}\left(\sum_{k=1}^{m} p_{k}(\Theta(A), \Theta(B))\right) x\right\|^{2}
\end{aligned}
$$


By (3.4) this inequality can only hold for $x=0$. Since $\Delta_{n}$ is open, by the Spectral Theorem for normal operators on Hilbert spaces we have $\Delta_{n} \subseteq$ $\rho(\Theta(N))$. The asserted inclusion now follows from

$$
\left\{z \in \mathbb{C}:\left|p_{j}(\operatorname{Re} z, \operatorname{Im} z)\right|>\left\|R_{j} R_{j}^{*}\right\| \cdot\left|\sum_{k=1}^{m} p_{k}(\operatorname{Re} z, \operatorname{Im} z)\right|\right\}=\bigcup_{n \in \mathbb{N}} \Delta_{n} .
$$

In the following let $\mathcal{I}$ be the ideal $\left\langle p_{1}, \ldots, p_{m}\right\rangle$ generated by the real definitizing polynomials $p_{1}, \ldots, p_{m}$ in the ring $\mathbb{C}[x, y]$. The variety $V(\mathcal{I})$ is the set of all common zeros $a=\left(a_{1}, a_{2}\right) \in \mathbb{C}^{2}$ of all $p \in \mathcal{I}$. Clearly, $V(\mathcal{I})$ coincides with the set of all $a \in \mathbb{C}^{2}$ such that $p_{1}\left(a_{1}, a_{2}\right)=\cdots=p_{m}\left(a_{1}, a_{2}\right)=0$. Denote by $V_{\mathbb{R}}(\mathcal{I})$ the set of all $a \in \mathbb{R}^{2}$, which belong to $V(\mathcal{I})$. It is convenient for our purposes, to consider $V_{\mathbb{R}}(\mathcal{I})$ as a subset of $\mathbb{C}$ :

$$
\begin{aligned}
V_{\mathbb{R}}(\mathcal{I}) & :=\{z \in \mathbb{C}: f(\operatorname{Re} z, \operatorname{Im} z)=0 & \text { for all } f \in \mathcal{I}\} \\
& =\left\{z \in \mathbb{C}: p_{k}(\operatorname{Re} z, \operatorname{Im} z)=0\right. & \text { for all } k \in\{1, \ldots, m\}\} .
\end{aligned}
$$

Corollary 3.6. Let $E$ denote the spectral measure of $\Theta(N)$. Then we have

$$
\begin{aligned}
R_{j} R_{j}^{*} E\left(\mathbb{C} \backslash V_{\mathbb{R}}(\mathcal{I})\right) & =E\left(\mathbb{C} \backslash V_{\mathbb{R}}(\mathcal{I})\right) R_{j} R_{j}^{*} \\
& =\int_{\mathbb{C} \backslash V_{\mathbb{R}}(\mathcal{I})} \frac{p_{j}(\operatorname{Re} z, \operatorname{Im} z)}{\sum_{k=1}^{m} p_{k}(\operatorname{Re} z, \operatorname{Im} z)} d E(z) .
\end{aligned}
$$

Proof. First note that the integral on the right hand side exists as a bounded operator, because by Lemma 3.5 we have $\left|p_{j}(\operatorname{Re} z, \operatorname{Im} z)\right| \leq\left\|R_{j} R_{j}^{*}\right\|$. $\left|\sum_{k=1}^{m} p_{k}(\operatorname{Re} z, \operatorname{Im} z)\right|$ for $z \in \sigma(\Theta(N))$. The first equality is known from Corollary 2.3.

Concerning the second equality, note that both sides vanish on the range of $E\left(V_{\mathbb{R}}(\mathcal{I})\right)$. Its orthogonal complement $\mathcal{Q}:=\operatorname{ran} E\left(\mathbb{C} \backslash V_{\mathbb{R}}(\mathcal{I})\right)$ is invariant under

$$
\int\left(\sum_{k=1}^{m} p_{k}(\operatorname{Re} z, \operatorname{Im} z)\right) d E(z)=\sum_{k=1}^{m} p_{k}(\Theta(A), \Theta(B)) .
$$

By Lemma 3.5 the restriction of this operator to $\mathcal{Q}$ is injective, and hence, has dense range in $\mathcal{Q}$. If $x$ belongs to this dense range, i.e. $x=\left(\sum_{k=1}^{m} p_{k}(\Theta(A)\right.$, $\Theta(B))) y$ with $y \in \mathcal{Q}$, then

$$
\begin{aligned}
& \int_{\mathbb{C} \backslash V_{\mathbb{R}}(\mathcal{I})} \frac{p_{j}(\operatorname{Re} z, \operatorname{Im} z)}{\sum_{k=1}^{m} p_{k}(\operatorname{Re} z, \operatorname{Im} z)} d E(z) x \\
& \quad=\int_{\mathbb{C} \backslash V_{\mathbb{R}}(\mathcal{I})} p_{j}(\operatorname{Re} z, \operatorname{Im} z) d E(z) y \\
& =p_{j}(\Theta(A), \Theta(B)) y=R_{j} R_{j}^{*}\left(\sum_{k=1}^{m} p_{k}(\Theta(A), \Theta(B))\right) y \\
& =R_{j} R_{j}^{*} x .
\end{aligned}
$$

By a density argument the second asserted equality of the present corollary holds true on $\mathcal{Q}$ and in turn on $\mathcal{H}$. 
Remark 3.7. In Proposition 3.3 the case that $p_{j}(A, B)=0$ for some $j$, or even for all $j$, is not excluded, and yields $\mathcal{H}_{j}=\{0\}, T_{j}=0$ and $R_{j}=0$ (in Lemma 3.4), or even $\mathcal{H}=\{0\}$ and $T=0$. Also the remaining results hold true, if we interpret $\rho(R)$ as $\mathbb{C}$ and $\sigma(R)$ as $\emptyset$ for the only possible linear operator $R=(0 \mapsto 0)$ on the vector space $\{0\}$.

\section{An Abstract Functional Calculus}

In this section let $\mathcal{K}$ be again a Krein space and let $N \in B(\mathcal{K})$ be a definitizable normal operator. Let $\mathcal{I}$ be the ideal in $\mathbb{C}[x, y]$, which is generated by all real definitizing polynomials. In order to increase readability, from now on we often write $p(z)$ short for $p(\operatorname{Re} z, \operatorname{Im} z)$ if $p \in \mathbb{C}[x, y]$ and $z \in \mathbb{C}$.

By the ascending chain condition for the ring $\mathbb{C}[x, y]$ (see for example [2], Theorem 7, Chap. 2, Sect. 5) $\mathcal{I}$ is generated by finitely many real definitizing polynomials $p_{1}, \ldots, p_{m}$, i.e. $\mathcal{I}=\left\langle p_{1}, \ldots, p_{m}\right\rangle$. In fact, if $\mathcal{I}$ would not be generated by finitely many real definitizing polynomials, then, in contrast to the ascending chain condition, we could find a sequence $\left(p_{n}\right)_{n \in \mathbb{N}}$ of such polynomials with $p_{n+1} \notin\left\langle p_{1}, \ldots, p_{n}\right\rangle$ for all $n \in \mathbb{N}$.

Using these polynomials $p_{1}, \ldots, p_{m}$, for $j=1, \ldots, m$ we define the spaces $\mathcal{H}_{j}, \mathcal{H}$, the operators $T_{j}, R_{j}, T$, and the spectral measures $E_{j}$ and $E$ of $\Theta_{j}(N)$ and $\Theta(N)$, respectively, as in the previous sections, where $\Theta_{j}, \Theta$ is defined in (2.1). Accordingly we define $\Xi_{j}$ and $\Xi$ as in (2.5).

Lemma 4.1. For any bounded and measurable $f: \sigma(\Theta(N)) \rightarrow \mathbb{C}$ and $j \in$ $\{1, \ldots, m\}$ we have

$$
\begin{aligned}
\Xi_{j}\left(\int f d E_{j}\right)= & \Xi\left(\int_{\sigma(\Theta(N)) \backslash V_{\mathbb{R}}(\mathcal{I})} f \frac{p_{j}}{\sum_{l=1}^{m} p_{l}} d E\right. \\
& \left.+R_{j} R_{j}^{*} \int_{\sigma(\Theta(N)) \cap V_{\mathbb{R}}(\mathcal{I})} f d E\right) .
\end{aligned}
$$

Proof. By (2.6) the left hand side coincides with

$$
\Xi\left(R_{j} R_{j}^{*} \int_{\sigma(\Theta(N)) \backslash V_{\mathbb{R}}(\mathcal{I})} f d E+R_{j} R_{j}^{*} \int_{\sigma(\Theta(N)) \cap V_{\mathbb{R}}(\mathcal{I})} f d E\right) .
$$

As $\int_{\sigma(\Theta(N)) \backslash V_{\mathbb{R}}(\mathcal{I})} f d E=E\left(\mathbb{C} \backslash V_{\mathbb{R}}(\mathcal{I})\right) \int_{\sigma(\Theta(N)) \backslash V_{\mathbb{R}}(\mathcal{I})} f d E$ Corollary 3.6 proves the asserted equality.

Lemma 4.2. Let $f, g: \sigma(\Theta(N)) \rightarrow \mathbb{C}$ be bounded and measurable, and let $r \in \mathbb{C}[x, y]$. For $j, k \in\{1, \ldots, m\}$ we then have

$$
r(A, B) \Xi_{j}\left(\int f d E_{j}\right)=\Xi_{j}\left(\int f d E_{j}\right) r(A, B)=\Xi_{j}\left(\int r f d E_{j}\right),
$$

and

$$
\begin{gathered}
\Xi_{j}\left(\int f d E_{j}\right) \Xi_{k}\left(\int g d E_{k}\right)=\Xi\left(\int f g \frac{p_{j} p_{k}}{\sum_{l=1}^{m} p_{l}} d E\right) \\
=\Xi_{j}\left(\int f g p_{k} d E_{j}\right)=\Xi_{k}\left(\int f g p_{j} d E_{k}\right) .
\end{gathered}
$$


Proof. By Lemma 5.11 in [5] we have

$$
\begin{aligned}
r(A, B) \Xi_{j}(D) & =\Xi_{j}(\Theta(r(A, B)) D)=\Xi_{j}\left(r\left(\Theta_{j}(A), \Theta_{j}(B)\right) D\right), \\
\Xi_{j}(D) r(A, B) & =\Xi_{j}\left(D \Theta_{j}(r(A, B))\right)=\Xi_{j}\left(\operatorname{Dr}\left(\Theta_{j}(A), \Theta_{j}(B)\right)\right)
\end{aligned}
$$

for $D \in\left(T^{+} T\right)^{\prime}$. For $D=\int f d E_{j}$ this implies (4.1).

According to (2.6) the expression in (4.2) coincides with

$$
\Xi\left(R_{j} R_{j}^{*} \int f d E\right) \Xi\left(R_{k} R_{k}^{*} \int g d E\right) .
$$

By Lemma 5.11 and Theorem 5.8 in [5], we also know that $\Xi\left(D_{1}\right) \Xi\left(D_{2}\right)=$ $\Xi\left(T^{+} T D_{1} D_{2}\right)=\Xi\left(\Theta\left(T T^{+}\right) D_{1} D_{2}\right)$, where (see Propositions 3.3 and (3.6))

$\Theta\left(T T^{+}\right)=\sum_{l=1}^{m} p_{l}(\Theta(A), \Theta(B))=\int \sum_{l=1}^{m} p_{l} d E=\left(\left(\int \sum_{l=1}^{m} p_{l} d E\right) E\left(\mathbb{C} \backslash V_{\mathbb{R}}(\mathcal{I})\right)\right)$.

Therefore, by Corollary 3.6 and by the fact, that $E\left(\mathbb{C} \backslash V_{\mathbb{R}}(\mathcal{I})\right)$ commutes with $\int_{\sigma(\Theta(N))} f d E,(4.2)$ can be written as

$$
\begin{aligned}
& \Xi\left(\left(\int \sum_{l=1}^{m} p_{l} d E\right)\left(\int \frac{p_{j}}{\sum_{l=1}^{m} p_{l}} d E\right)\left(\int f d E\right)\left(\int \frac{p_{k}}{\sum_{l=1}^{m} p_{l}} d E\right)\left(\int g d E\right)\right) \\
& \quad=\Xi\left(\int f g \frac{p_{j} p_{k}}{\sum_{l=1}^{m} p_{l}} d E\right) .
\end{aligned}
$$

The remaining equalities follow from Lemma 4.1 since the respective integrands vanish on $V_{\mathbb{R}}(\mathcal{I})$.

Lemma 4.3. For any bounded and measurable $f: \sigma(\Theta(N)) \rightarrow \mathbb{C}$ and $j \in$ $\{1, \ldots, m\}$ the operator $\Xi_{j}\left(\int f d E_{j}\right)$ belongs to $\left\{N, N^{+}\right\}^{\prime \prime}$.

Proof. Take $C \in\left\{N, N^{+}\right\}^{\prime}=\{A, B\}^{\prime} \subseteq \bigcap_{j=1, \ldots, m}\left(T_{j} T_{j}^{+}\right)^{\prime}$; see (3.3). From Lemma 5.11 in [5] we conclude

$$
C \Xi_{j}\left(\int f d E_{j}\right)=\Xi_{j}\left(\Theta_{j}(C)\left(\int f d E_{j}\right)\right) .
$$

Since $\Theta_{j}$ is a homomorphism, $\Theta_{j}(C)$ commutes with $\Theta_{j}(N)$ and, in turn, with $\int_{\sigma\left(\Theta_{j}(N)\right)} f d E_{j}$. Hence, employing Lemma 5.11 in [5] once more, the above expression coincides with

$$
\Xi_{j}\left(\left(\int f d E_{j}\right) \Theta_{j}(C)\right)=\Xi_{j}\left(\int f d E_{j}\right) C .
$$

In order to have a better picture of what is going on, the tupel $\left(r, f_{1}, \ldots\right.$, $f_{m}$ ) appearing in the subsequent definition should be imagined as the function $r+p_{1} \cdot f_{1}+\ldots p_{m} \cdot f_{m}$ with a special behaviour at the points $\sigma(\Theta(N)) \cap V_{\mathbb{R}}(\mathcal{I})$.

Definition 4.4. Denoting by $\mathfrak{B}(\sigma(\Theta(N)))$ the *-algebra of complex valued, bounded and measurable functions on $\sigma(\Theta(N))$, for $\left(r, f_{1}, \ldots, f_{m}\right) \in \mathcal{R}:=$ $\mathbb{C}[x, y] \times \mathfrak{B}(\sigma(\Theta(N))) \times \cdots \times \mathfrak{B}(\sigma(\Theta(N)))$ we set

$$
\Psi\left(r, f_{1}, \ldots, f_{m}\right):=r(A, B)+\sum_{k=1}^{m} \Xi_{k}\left(\int f_{k} d E_{k}\right) .
$$


By $\mathcal{N}$ we denote the set of all $\left(r, f_{1}, \ldots, f_{m}\right) \in \mathcal{R}$ such that

$$
r+\sum_{k=1}^{m} f_{k} p_{k}=0 \quad \text { on } \quad \sigma(\Theta(N)) \backslash V_{\mathbb{R}}(\mathcal{I})
$$

and such that there exist $u_{1}, \ldots, u_{m} \in \mathbb{C}[x, y]$ with $r=\sum_{k=1}^{m} u_{k} p_{k}$ and $\left(f_{j}+u_{j}\right)(z)=0$ for $j=1, \ldots, m, z \in V_{\mathbb{R}}(\mathcal{I}) \cap \sigma(\Theta(N))$.

Remark 4.5. Obviously, $\Psi$ is linear. From $\Xi_{j}\left(D^{*}\right)=\Xi_{j}(D)^{+}$we easily deduce $\Psi\left(r^{\#}, \overline{f_{1}}, \ldots, \overline{f_{m}}\right)=\Psi\left(r, f_{1}, \ldots, f_{m}\right)^{*}$. Moreover, $\mathcal{N}$ constitutes a linear subspace of $\mathcal{R}$ invariant under.\# $:\left(r, f_{1}, \ldots, f_{m}\right) \mapsto\left(r^{\#}, \overline{f_{1}}, \ldots, \overline{f_{m}}\right)$.

Lemma 4.6. If $\left(r, f_{1}, \ldots, f_{m}\right) \in \mathcal{N}$, then $\Psi\left(r, f_{1}, \ldots, f_{m}\right)=0$.

Proof. Due to (3.5) $r=\sum_{k=1}^{m} u_{k} p_{k}$ implies

$$
r(A, B)=\sum_{k=1}^{m} p_{k}(A, B) u_{k}(A, B)=\sum_{k=1}^{m} \Xi_{k}\left(u_{k}\left(\Theta_{k}(A), \Theta_{k}(B)\right)\right) .
$$

From this and Lemma 4.1 we obtain

$$
\begin{gathered}
\Psi\left(r, f_{1}, \ldots, f_{m}\right)=\sum_{k=1}^{m} \Xi_{k}\left(\int\left(f_{k}+u_{k}\right) d E_{k}\right)= \\
\Xi\left(\int_{\sigma(\Theta(N)) \backslash V_{\mathbb{R}}(\mathcal{I})} \sum_{k=1}^{m} \frac{f_{k} p_{k}+u_{k} p_{k}}{\sum_{l=1}^{m} p_{l}} d E+\sum_{k=1}^{m} R_{k} R_{k}^{*} \int_{\sigma(\Theta(N)) \cap V_{\mathbb{R}}(\mathcal{I})}\left(f_{k}+u_{k}\right) d E\right),
\end{gathered}
$$

which by the definition of $\mathcal{N}$ equals to 0 .

Lemma 4.7. For $\left(r, f_{1}, \ldots, f_{m}\right),\left(s, g_{1}, \ldots, g_{m}\right) \in \mathcal{R}$ have $\Psi\left(r, f_{1}, \ldots, f_{m}\right) \Psi\left(s, g_{1}, \ldots, g_{m}\right)$

$$
\begin{aligned}
& =\Psi\left(r s, r g_{1}+s f_{1}+f_{1} \sum_{k=1}^{m} g_{k} p_{k}, \ldots, r g_{m}+s f_{m}+f_{m} \sum_{k=1}^{m} g_{k} p_{k}\right) \\
& =\Psi\left(r s, r g_{1}+s f_{1}+g_{1} \sum_{k=1}^{m} f_{k} p_{k}, \ldots, r g_{m}+s f_{m}+g_{m} \sum_{k=1}^{m} f_{k} p_{k}\right) .
\end{aligned}
$$

Proof. By Lemma 4.2 we have

$$
\begin{aligned}
& \Psi\left(r, f_{1}, \ldots, f_{m}\right) \Psi\left(s, g_{1}, \ldots, g_{m}\right)=r(A, B) s(A, B) \\
& \quad+\sum_{k=1}^{m} r(A, B) \Xi_{k}\left(\int g_{k} d E_{k}\right)+\sum_{j=1}^{m} \Xi_{j}\left(\int f_{j} d E_{j}\right) s(A, B) \\
& \quad+\sum_{j, k=1}^{m} \Xi_{j}\left(\int f_{j} d E_{j}\right) \Xi_{k}\left(\int g_{k} d E_{k}\right) \\
& =(r s)(A, B)+\sum_{k=1}^{m} \Xi_{k}\left(\int r g_{k} d E_{k}\right)+\sum_{j=1}^{m} \Xi_{j}\left(\int s f_{j} d E_{j}\right) \\
& \quad+\sum_{j=1}^{m} \Xi_{j}\left(\sum_{k=1}^{m} \int f_{j} g_{k} p_{k} d E_{j}\right)
\end{aligned}
$$


where this last term can also be written as

$$
\sum_{j=1}^{m} \Xi_{j}\left(\sum_{k=1}^{m} \int f_{k} g_{j} p_{k} d E_{j}\right) .
$$

We provide $\mathcal{R}$ with a multiplication:

$$
\begin{aligned}
& \left(r, f_{1}, \ldots, f_{m}\right) \cdot\left(s, g_{1}, \ldots, g_{m}\right):= \\
& \quad\left(r s, r g_{1}+s f_{1}+f_{1} \sum_{j=1}^{m} g_{j} p_{j}, \cdots, r g_{m}+s f_{m}+f_{m} \sum_{j=1}^{m} g_{j} p_{j}\right) .
\end{aligned}
$$

Remark 4.8. Obviously, · is bilinear and compatible with .\# as defined in Remark 4.5. It is elementary to check its associativity.

Moreover, for $\left(r, f_{1}, \ldots, f_{m}\right) \in \mathcal{N}$ and $\left(s, g_{1}, \ldots, g_{m}\right) \in \mathcal{R}$ we have $r s+\sum_{j=1}^{m} p_{j}\left(r g_{j}+s f_{j}+f_{j} \sum_{k=1}^{m} g_{k} p_{k}\right)=\left(r+\sum_{j=1}^{m} f_{j} p_{j}\right)\left(s+\sum_{k=1}^{m} g_{k} p_{k}\right)=0$ on $\mathbb{C} \backslash V_{\mathbb{R}}(\mathcal{I})$. For the corresponding $u_{1}, \ldots, u_{m} \in \mathbb{C}[x, y]$ with $r=\sum_{j=1}^{m} u_{j} p_{j}$ and $\left(f_{j}+u_{j}\right)(z)=0$ for all $z \in V_{\mathbb{R}}(\mathcal{I})$ we have $r s=\sum_{j=1}^{m}\left(u_{j} s\right) p_{j}$ and

$$
r g_{j}+s f_{j}+f_{j} \sum_{k=1}^{m} g_{k} p_{k}+u_{j} s=r g_{j}+f_{j} \sum_{k=1}^{m} g_{k} p_{k}=0
$$

on $V_{\mathbb{R}}(\mathcal{I})$ since $r$ and the $p_{j}$ vanish there. Hence, $\mathcal{N}$ is a right ideal. Similarly, one shows that it is also a left ideal. Finally, the commutator

$$
\begin{gathered}
\left(r, f_{1}, \ldots, f_{m}\right) \cdot\left(s, g_{1}, \ldots, g_{m}\right)-\left(s, g_{1}, \ldots, g_{m}\right) \cdot\left(r, f_{1}, \ldots, f_{m}\right)= \\
\left(0, \sum_{j=1}^{m}\left(f_{1} g_{j}-g_{1} f_{j}\right) p_{j}, \ldots, \sum_{j=1}^{m}\left(f_{m} g_{j}-g_{m} f_{j}\right) p_{j}\right)
\end{gathered}
$$

belongs to $\mathcal{N}$. Consequently, $\mathcal{R} / \mathcal{N}$ is a commutative $*$-algebra.

Gathering the previous results we obtain the final result of the present section.

Theorem 4.9. $\Psi / \mathcal{N}:\left(r, f_{1}, \ldots, f_{m}\right)+\mathcal{N} \mapsto \Psi\left(r, f_{1}, \ldots, f_{m}\right)$ is a well-defined *-homomorphism from $\mathcal{R} / \mathcal{N}$ into $\left\{N, N^{+}\right\}^{\prime \prime} \subseteq B(\mathcal{K})$.

\section{Algebra of Zero-Dimensional Ideals}

By the Noether-Lasker Theorem (see for example [2], Theorem 7, Chap. 4, Sect. 7) any ideal $\mathcal{I}$ in $\mathbb{C}[x, y]$ admits a minimal primary decomposition

$$
\mathcal{I}=Q_{1} \cap \cdots \cap Q_{l},
$$

$Q_{j}$ being a primary ideal means that $f g \in Q_{j}$ implies $f \in Q_{j}$ or $g^{k} \in Q_{j}$ for some $k \in \mathbb{N}$, and minimal means that $Q_{j} \nsupseteq \bigcap_{i \neq j} Q_{i}$ for all $j=1, \ldots, l$, and $P_{j} \neq P_{i}$ for $i \neq j$, where $P_{j}$ denotes the radical

$$
\sqrt{Q_{j}}:=\left\{f \in \mathbb{C}[x, y]: f^{k} \in Q_{j} \text { for some } k \in \mathbb{N}\right\} .
$$


For an ideal $\mathcal{I}$ in $\mathbb{C}[x, y]$ such a decomposition is in general not unique. Nevertheless, the First Uniqueness Theorem on minimal primary decompositions states that the number $l \in \mathbb{N}$ and the radicals $P_{1}, \ldots, P_{l}$ are uniquely determined by $\mathcal{I}$; see for example [1], Theorem 8.55 on page 362 . Moreover, the Second Uniqueness Theorem on minimal primary decompositions says that if $Q_{1}^{\prime} \cap \cdots \cap Q_{l}^{\prime}=\mathcal{I}=Q_{1} \cap \cdots \cap Q_{l}$ are minimal primary decompositions ordered such that $P_{j}=\sqrt{Q_{j}}=\sqrt{Q_{j}^{\prime}}$ for $j=1, \ldots, l$ and if $P_{k}$ is minimal in $\left\{P_{1}, \ldots, P_{l}\right\}$ with respect to $\subseteq$, then $Q_{k}^{\prime}=Q_{k}$; see for example [1], Theorem 8.56 on page 364 .

Assume now that $\mathcal{I}$ is a zero-dimensional ideal in $\mathbb{C}[x, y]$, i.e.

$$
\operatorname{dim} \mathbb{C}[x, y] / \mathcal{I}<\infty .
$$

For necessary and sufficient conditions see for example [1], Theorem 6.54 and Corollary 6.56 on pages 274 and 275 and [3], pages 39 and 40. Let (5.1) be a minimal primary decomposition. Then any $Q_{j}$, and in turn $P_{j} \supseteq Q_{j}$, is also zero-dimensional. In particular, $\mathbb{C}[x, y] / P_{j}$ is a finite integral domain, and hence, a field. In turn, the radicals $P_{1}, \ldots, P_{l}$ of $Q_{1}, \ldots, Q_{l}$ are maximal ideals. By [2], Theorem 11, Chap. 4, Sect. 5, this means that the $P_{j}$ are generated by $x-a_{x, j}, y-a_{y, j}$, i.e. $P_{j}=\left\langle x-a_{x, j}, y-a_{y, j}\right\rangle$, for pairwise distinct $a_{j}=\left(a_{x, j}, a_{y, j}\right) \in \mathbb{C}^{2}$. Consequently, any $P_{k}$ is minimal in $\left\{P_{1}, \ldots, P_{l}\right\}$, and by what was said above, (5.1) is the unique minimal primary decomposition of $\mathcal{I}$.

By Hilbert's Nullstellensatz (see for example [2], Theorem 2, Chap. 4, Sect. 1) the set $V\left(Q_{j}\right)$ of common zeros in $\mathbb{C}^{2}$ of all $f \in Q_{j}$ coincides with $V\left(P_{j}\right)=\left\{a_{j}\right\}$. By [2], Theorem 7, Chap. 4, Sect. 3, we also have

$$
V(\mathcal{I})=V\left(Q_{1}\right) \cup \cdots \cup V\left(Q_{l}\right)=\left\{a_{1}, \ldots, a_{l}\right\} .
$$

Since $V\left(Q_{j}+Q_{i}\right)=V\left(Q_{j}\right) \cap V\left(Q_{i}\right)=\left\{a_{j}\right\} \cap\left\{a_{i}\right\}=\emptyset$ (see [2], Theorem 4, Chap. 4, Sect. 3) for $i \neq j$, the weak Nullstellensatz (see for example [2], Theorem 1, Chap. 4, Sect. 1) yields $Q_{j}+Q_{i}=\mathbb{C}[x, y]$. By the Chinese Remainder Theorem the mapping

$$
\theta:\left\{\begin{array}{l}
\mathbb{C}[x, y] / \mathcal{I} \rightarrow\left(\mathbb{C}[x, y] / Q_{1}\right) \times \cdots \times\left(\mathbb{C}[x, y] / Q_{l}\right), \\
x+\mathcal{I} \mapsto\left(x+Q_{1}, \ldots, x+Q_{l}\right)
\end{array}\right.
$$

constitutes an isomorphism. Moreover,

$$
\mathcal{I}=Q_{1} \cap \cdots \cap Q_{l}=Q_{1} \cdot \ldots \cdot Q_{l} .
$$

Remark 5.1.

1. Since the ring $\mathbb{C}[x, y] / Q_{j}$ is finite dimensional, its invertible elements $f+Q_{j}$ are exactly those, for which $f g \in Q_{j}$ implies $g \in Q_{j} . Q_{j}$ being primary this is equivalent to $f \notin P_{j}$. Hence, $f+Q_{j}$ is invertible in $\mathbb{C}[x, y] / Q_{j}$ if and only if $f\left(a_{j}\right) \neq 0$.

2. As $\sqrt{Q_{j}}=P_{j}$ we have $\left(x-a_{x, j}\right)^{m},\left(y-a_{y, j}\right)^{n} \in Q_{j}$ for sufficiently large $m, n \in \mathbb{N}$. Therefore, the ideal $P_{j} \cdot Q_{j}$ contains $\left(x-a_{x, j}\right)^{m+1}$ and $\left(y-a_{y, j}\right)^{n+1}$. Thus, $P_{j} \cdot Q_{j}$ is also zero-dimensional and $\sqrt{P_{j} \cdot Q_{j}}=P_{j}$. 
Definition 5.2. For $a \in V(\mathcal{I})$ we set by $Q(a):=Q_{j}$ and $P(a):=P_{j}$, where $j$ is such that $a=a_{j}$. By $d_{x}(a)\left(d_{y}(a)\right)$ we denote the smallest natural number $m(n)$ such that $\left(x-a_{x}\right)^{m} \in Q(a)\left(\left(y-a_{y}\right)^{n} \in Q(a)\right)$. Moreover, we set

$$
\mathcal{A}(a):=\mathbb{C}[x, y] /(P(a) \cdot Q(a)) \text { and } \mathcal{B}(a):=\mathbb{C}[x, y] / Q(a) .
$$

for $a \in V(\mathcal{I})$.

Since $P(a) \cdot Q(a)$ and $Q(a)$ are ideals with finite codimension satisfying $P(a) \cdot Q(a) \subseteq Q(a), \mathcal{A}(a)$ and $\mathcal{B}(a)$ are finite dimensional algebras with $\operatorname{dim} \mathcal{A}(a) \geq \operatorname{dim} \mathcal{B}(a)$.

Remark 5.3. Assume that $\mathcal{I}$ is invariant under .\#, where $f^{\#}(x, y):=\overline{f(\bar{x}, \bar{y})}$. This is for sure the case if $\mathcal{I}$ is generated by real polynomial $p_{1}, \ldots, p_{m}$. Then $V(\mathcal{I}) \subseteq \mathbb{C}^{2}$ is invariant under $(z, w) \mapsto(z, w)^{\#}:=(\bar{z}, \bar{w})$. Moreover, it is elementary to check that with $Q$ also $Q^{\#}$ is a primary ideal. Hence, with $\mathcal{I}=Q_{1} \cap \cdots \cap Q_{l}$ also $\mathcal{I}=\mathcal{I}^{\#}=Q_{1}^{\#} \cap \cdots \cap Q_{l}^{\#}$ is a minimal primary decomposition. By the uniqueness of the minimal primary decomposition for our zero dimensional ideal $\mathcal{I}$ one has $Q(a)^{\#}=Q\left(a^{\#}\right)$ for all $a \in V(\mathcal{I})$.

Consequently, $f \mapsto f^{\#}$ induces a conjugate linear bijection from $\mathcal{A}(a)$ $(\mathcal{B}(a))$ onto $\mathcal{A}\left(a^{\#}\right)\left(\mathcal{B}\left(a^{\#}\right)\right)$.

For the following note that if we conversely start with primary and zero-dimensional ideals $Q_{1}, \ldots, Q_{l}$ with $\sqrt{Q_{i}} \neq \sqrt{Q_{j}}$ for $i \neq j$, then $\mathcal{I}:=$ $Q_{1} \cap \cdots \cap Q_{l}$ is also zero-dimensional, and by the above mentioned uniqueness statement, $Q_{1} \cap \cdots \cap Q_{l}$ is indeed the unique minimal primary decomposition of $\mathcal{I}$.

Proposition 5.4. Let $\mathcal{I}$ be a zero-dimensional ideal in $\mathbb{C}[x, y]$ which is generated by $p_{1}, \ldots, p_{m}$, and let $\mathcal{I}=\bigcap_{a \in V(\mathcal{I})} Q(a)$ be its unique primary decomposition. Assume that $W$ is a subset of $V(\mathcal{I})$. Then

$$
\mathcal{J}:=\bigcap_{a \in V(\mathcal{I}) \backslash W} Q(a) \cap \bigcap_{a \in W}(P(a) \cdot Q(a))
$$

is also a zero-dimensional ideal satisfying $\mathcal{J} \subseteq \mathcal{I}$. The mapping

$$
\psi:\left\{\begin{array}{l}
\mathbb{C}[x, y] / \mathcal{J} \underset{a \in V(\mathcal{I}) \backslash W}{\rightarrow}(\mathbb{C}[x, y] / Q(a)) \times \underset{a \in W}{\times}(\mathbb{C}[x, y] /(P(a) \cdot Q(a))), \\
x+\mathcal{J} \quad \mapsto\left((x+Q(a))_{a \in V(\mathcal{I}) \backslash W},(x+(P(a) \cdot Q(a)))_{a \in W}\right)
\end{array}\right.
$$

is an isomorphism, and any $p \in \mathcal{J}$ can be written in the form $p=\sum_{j} u_{j} p_{j}$, where $u_{j}(a)=0$ for all $a \in W$.

Proof. We already mentioned that $P(a) \cdot Q(a)$ is zero-dimensional with $\sqrt{P(a) \cdot Q(a)}=P(a)$ and that the intersection $\mathcal{J}=\bigcap_{a \in V(\mathcal{I}) \backslash W} Q(a) \cap$ $\bigcap_{a \in W} P(a) \cdot Q(a)$ is the unique primary decomposition of the zero-dimensional $\mathcal{J}$. The isomorphism property of $\psi$ is a special case of the corresponding fact 
concerning $\theta$; see (5.2). We also have

$$
\begin{aligned}
\mathcal{J} & =\prod_{a \in V(\mathcal{I}) \backslash W} Q(a) \cdot \prod_{a \in W} P(a) \cdot Q(a)=\prod_{a \in V(\mathcal{I})} Q(a) \cdot \prod_{a \in W} P(a) \\
& =\mathcal{I} \cdot \prod_{a \in W} P(a)=\left\langle p_{1} \cdot \prod_{a \in W} P(a), \ldots, p_{m} \cdot \prod_{a \in W} P(a)\right\rangle .
\end{aligned}
$$

This means that any $p \in \mathcal{J}$ has a representation $p=\sum_{j} u_{j} p_{j}$ with $u_{j} \in$ $\prod_{a \in W} P(a)=\bigcap_{a \in W} P(a)$. Hence, $u_{j}(a)=0$ for all $a \in W$.

Example 5.5. Assume that $\mathcal{I}$ is generated by two polynomial $p_{1}, p_{2} \in \mathbb{C}[x, y]$ such that $p_{1}$ only depend on $x$ and $p_{2}$ only depends on $y$. The set $V(\mathcal{I})$ of common zeros of $\mathcal{I}$, or equivalently of $p_{1}$ and $p_{2}$, in $\mathbb{C}^{2}$ then consists of all points of the form $(z, w)$, where $z \in \mathbb{C}$ is a zero of $p_{1}$ and $w \in \mathbb{C}$ is a zero of $p_{2}$, i.e. $V(\mathcal{I})=p_{1}^{-1}\{0\} \times p_{2}^{-1}\{0\}$. For $z \in p_{1}^{-1}\{0\}$ by $\mathfrak{d}_{1}(z)$ we denote $p_{1}$ 's multiplicity of the zero $z$, and for $w \in p_{2}^{-1}\{0\}$ by $\mathfrak{d}_{2}(w)$ we denote $p_{2}$ 's multiplicity of the zero $w$.

Given $p \in \mathbb{C}[x, y]$ we can apply polynomial division in one variable twice, once with respect to $x$ and once with respect to $y$, on order to see that

$$
p(x, y)=p_{1}(x) \cdot u(x, y)+p_{2}(y) \cdot v(x, y)+q(x, y)
$$

with $u, v, q \in \mathbb{C}[x, y]$ such that the degree of $q$, seen as a polynomial on $x$, is less then the degree of $p_{1}$, and such that the degree of $q$, seen as a polynomial on $y$, is less then the degree of $p_{2}$; see Lemma 4.8 in [4]. Hence, $\mathcal{I}$ is zerodimensional. Moreover, writing $p_{1}(x)$ and $p_{2}(y)$ as products of linear factors, it follows that $p \in \mathcal{I}$ if and only if

$$
p \in\left\langle(x-z)^{\mathfrak{d}_{1}(z)},(y-w)^{\mathfrak{d}_{2}(w)}\right\rangle=: Q((z, w)),
$$

for all $z \in p_{1}^{-1}\{0\}, w \in p_{2}^{-1}\{0\}$. Since $Q((z, w))$ is a primary ideal in $\mathbb{C}[x, y]$,

$$
\mathcal{I}=\bigcap_{(z, w) \in p_{1}^{-1}\{0\} \times p_{2}^{-1}\{0\}} Q((z, w))
$$

is the minimal primary decomposition of $\mathcal{I}$. For the respective radicals we have $P((z, w))=\langle x-z, y-w\rangle$. Moreover, $P((z, w)) \cdot Q((z, w))$ coincides with

$$
\left\langle(x-z)^{\mathfrak{d}_{1}(z)+1},(x-z)^{\mathfrak{d}_{1}(z)}(y-w),(x-z)(y-w)^{\mathfrak{d}_{2}(w)},(y-w)^{\mathfrak{d}_{2}(w)+1}\right\rangle .
$$

Therefore, $\mathcal{A}((z, w))=\mathbb{C}[x, y] /(P((z, w)) \cdot Q((z, w)))$ is isomorphic to $\mathcal{A}_{\mathfrak{d}_{1}(z), \mathfrak{d}_{2}(w)}$ and $\mathcal{B}((z, w))=\mathbb{C}[x, y] / Q((z, w))$ is isomorphic to $\mathcal{B}_{\mathfrak{d}_{1}(z), \mathfrak{d}_{2}(w)}$ as introduced in Definition 4.1, [4].

\section{Function Classes}

In the present section we make the same assumptions and use the same notation as in Sect. 4. In addition, we assume that the ideal $\mathcal{I}$ generated by all real definitizing polynomials is zero-dimensional. We fix real, definitizing polynomials $p_{1}, \ldots, p_{m}$ which generate $\mathcal{I}$. For the zero-dimensional $\mathcal{I}$ we apply the 
same notation as in the previous section. The variety $V(\mathcal{I})=\left\{a_{1}, \ldots, a_{l}\right\} \subseteq$ $\mathbb{C}^{2}$ of common zeros of all $f \in \mathcal{I}$ will be split up as

$$
V(\mathcal{I})=\underbrace{\left(V(\mathcal{I}) \cap \mathbb{R}^{2}\right)}_{=V_{\mathbb{R}}(\mathcal{I})} \dot{\cup}\left(V(\mathcal{I}) \backslash \mathbb{R}^{2}\right),
$$

where we consider $V_{\mathbb{R}}(\mathcal{I})$ as a subset of $\mathbb{C}$; see (3.6).

Definition 6.1. By $\mathcal{M}_{N}$ we denote the set of functions $\phi$ defined on

$$
\underbrace{\left(\sigma(\Theta(N)) \cup V_{\mathbb{R}}(\mathcal{I})\right)}_{\subseteq \mathbb{C}} \dot{\cup} \underbrace{\left(V(\mathcal{I}) \backslash \mathbb{R}^{2}\right)}_{\subseteq \mathbb{C}^{2}}
$$

with $\phi(z) \in \mathbb{C}$ for $z \in \sigma(\Theta(N)) \backslash V_{\mathbb{R}}(\mathcal{I}), \phi(z) \in \mathcal{A}(z)$ for $z \in V_{\mathbb{R}}(\mathcal{I})$, and $\phi(z) \in \mathcal{B}(z)$ for $z \in V(\mathcal{I}) \backslash \mathbb{R}^{2}$.

We provide $\mathcal{M}_{N}$ pointwise with scalar multiplication, addition and multiplication. We also define a conjugate linear involution . $\#$ on $\mathcal{M}_{N}$ by

$$
\begin{array}{ll}
\phi^{\#}(z):=\overline{\phi(z)} & \text { for } z \in \sigma(\Theta(N)) \backslash V_{\mathbb{R}}(\mathcal{I}), \\
\phi^{\#}(z):=\phi(z)^{\#} & \text { for } z \in V_{\mathbb{R}}(\mathcal{I}) \\
\phi^{\#}(\xi, \eta):=\phi(\bar{\xi}, \bar{\eta})^{\#} & \text { for }(\xi, \eta) \in V(\mathcal{I}) \backslash \mathbb{R}^{2} .
\end{array}
$$

With the operations introduced above $\mathcal{M}_{N}$ is a commutative $*$-algebra as can be verified in a straight forward manner; see Remark 5.3.

Definition 6.2. Let $f: \operatorname{dom} f \rightarrow \mathbb{C}$ be a function with $\operatorname{dom} f \subseteq \mathbb{C}^{2}$ such that $\tau\left(\sigma(\Theta(N)) \cup V_{\mathbb{R}}(\mathcal{I})\right) \subseteq \operatorname{dom} f$, where $\tau: \mathbb{C} \rightarrow \mathbb{C}^{2},(x+i y) \mapsto(x, y)$, such that $f \circ \tau$ is sufficiently smooth - more exactly, at least $d_{x}(z)+d_{y}(z)-1$ times continuously differentiable - on a sufficiently small open neighbourhood $z$ for each $z \in V_{\mathbb{R}}(\mathcal{I})$, and such that $f$ is holomorphic on an open neighbourhood of $V(\mathcal{I}) \backslash \mathbb{R}^{2}\left(\subseteq \mathbb{C}^{2}\right)$.

Then $f$ can be considered as an element $f_{N}$ of $\mathcal{M}_{N}$ by setting $f_{N}(z):=$ $f \circ \tau(z)$ for $z \in \sigma(\Theta(N)) \backslash V_{\mathbb{R}}(\mathcal{I})$, by

$$
\begin{aligned}
f_{N}(z):= & \left.\sum_{(k, l) \in J(z)} \frac{1}{k ! l !} \frac{\partial^{k+l}}{\partial a^{k} \partial b^{l}} f \circ \tau(a+i b)\right|_{a+i b=z} \\
& \cdot(x-\operatorname{Re} z)^{k}(y-\operatorname{Im} z)^{l}+(P(z) \cdot Q(z)) \in \mathcal{A}(z)
\end{aligned}
$$

for $z \in V_{\mathbb{R}}(\mathcal{I})$, where

$$
J(z)=\left(\left\{0, \ldots, d_{x}(z)-1\right\} \times\left\{0, \ldots, d_{y}(z)-1\right\}\right) \cup\left\{\left(d_{x}(z), 0\right),\left(0, d_{y}(z)\right)\right\},
$$


and by

$$
\begin{aligned}
f_{N}(\xi, \eta):= & \left.\sum_{k=0}^{d_{x}(\xi, \eta)-1} \sum_{l=0}^{d_{y}(\xi, \eta)-1} \frac{1}{k ! l !} \frac{\partial^{k+l}}{\partial z^{k} \partial w^{l}} f(z, w)\right|_{(z, w)=(\xi, \eta)} \\
& \cdot(x-\xi)^{k}(y-\eta)^{l}+Q((\xi, \eta)) \in \mathcal{B}((\xi, \eta))
\end{aligned}
$$

for $(\xi, \eta) \in V(\mathcal{I}) \backslash \mathbb{R}^{2}$.

Remark 6.3. By the Leibniz rule $f \mapsto f_{N}$ is compatible with multiplication. Obviously, it is also compatible with addition and scalar multiplication. If we define for a function $f$ as in Definition 6.2 the function $f^{\#}$ by $f^{\#}(z, w)=$ $\overline{f(\bar{z}, \bar{w})},(z, w) \in \operatorname{dom} f$, then we also have $\left(f^{\#}\right)_{N}=\left(f_{N}\right)^{\#}$.

Remark 6.4. A special type of functions $f$ as in Definition 6.2 are polynomials in two variables, i.e. $f \in \mathbb{C}[x, y]$. Since for $z \in V_{\mathbb{R}}(\mathcal{I})$ and $(k, l) \notin J(z)$ we have $(x-\operatorname{Re} z)^{k}(y-\operatorname{Im} z)^{l} \in P(z) \cdot Q(z)$,

$$
f_{N}(z)=f+(P(z) \cdot Q(z)) \in \mathcal{A}(z) .
$$

Similarly, $f_{N}(\xi, \eta)=f+Q((\xi, \eta)) \in \mathcal{B}((\xi, \eta))$ for $(\xi, \eta) \in V(\mathcal{I}) \backslash \mathbb{R}^{2}$.

In particular, for $f=\mathbb{1}$ the element $f_{N}(z)$ is the multiplicative unite in $\mathcal{A}(z)$ or $\mathcal{B}(z)$ for all $z \in\left(\sigma(\Theta(N)) \cup V_{\mathbb{R}}(\mathcal{I})\right) \dot{\cup}\left(V(\mathcal{I}) \backslash \mathbb{R}^{2}\right)$.

For the following recall for example from [2], Theorem 4, Chap. 2, Sect. 5 , that any ideal in $\mathbb{C}[x, y]$ always has a finite number of generators.

Definition 6.5. For any $w \in \sigma(\Theta(N)) \cap V_{\mathbb{R}}(\mathcal{I})$ such that $w$ is not isolated in $\sigma(\Theta(N))$ let $h_{1}, \ldots, h_{n}$ be generators of the ideal $Q(w)$. For a sufficiently small neighbourhood $U(w)$ of $w$ let $\chi_{Q(w)}: U(w) \backslash\{w\} \rightarrow[0,+\infty)$ be

$$
\chi_{Q(w)}(z):=\max _{j=1, \ldots, n}\left|h_{j}(z)\right|,
$$

where $h_{j}(z)$, as usually, stands for $h_{j}(\operatorname{Re} z, \operatorname{Im} z)$.

Remark 6.6. Since $w$ is a common zero of all $h \in Q(w)$, we have $\chi_{Q(w)}(z) \rightarrow 0$ for $z \rightarrow w$. Moreover, for any $h \in Q(w)$ the fact, that $h_{1}, \ldots, h_{n}$ are generators of $Q(w)$, yields $h(z)=O\left(\chi_{Q(w)}(z)\right)$ as $z \rightarrow w$. This is particularly true for $h \in \mathcal{I}$.

Moreover, if $\chi_{Q(w)}^{\prime}$ is defined in a similar manner starting with generators $h_{1}^{\prime}, \ldots, h_{n^{\prime}}^{\prime}$, then $\chi_{Q(w)}^{\prime}(z)=O\left(\chi_{Q(w)}\right)(z)$ and $\chi_{Q(w)}(z)=O\left(\chi_{Q(w)}^{\prime}\right)(z)$ as $z \rightarrow w$. Hence, as far as it concerns the order of growth towards $w$, the expression $\chi_{Q(w)}$ does not depend on the actually chosen generators.

Finally, for $h \in Q(w)$ the polynomial

$$
g_{h}(x, y):=h(x, y) \cdot \prod_{a \in V(\mathcal{I}), a \neq(\operatorname{Re} w, \operatorname{Im} w)}\left(x-a_{x}\right)^{\epsilon_{x}(a)}\left(y-a_{y}\right)^{\epsilon_{y}(a)}
$$

belongs to $\mathcal{I}$, where $\epsilon_{x}(a)=d_{x}(a), \epsilon_{y}(a)=0$ or $\epsilon_{x}(a)=0, \epsilon_{y}(a)=d_{y}(a)$ depending on whether $a_{y}=\operatorname{Im} w$ or $a_{y} \neq \operatorname{Im} w$; see Definition 5.2. Since $\mathcal{I}$ is generated by $p_{1}, \ldots, p_{m}$, we have $g_{h}(z)=O\left(\max _{j=1, \ldots, m}\left|p_{j}(z)\right|\right)$ and, in turn, $h(z)=O\left(\max _{j=1, \ldots, m}\left|p_{j}(z)\right|\right)$ as $z \rightarrow w$. Applying this to $h=h_{j}$, we obtain $\chi_{Q(w)}(z)=O\left(\max _{j=1, \ldots, m}\left|p_{j}(z)\right|\right)$. Hence, as far as it concerns the 
order of growth towards $w$, the expression $\chi_{Q(w)}$ could also be defined as $\max _{j=1, \ldots, m}\left|p_{j}(z)\right|$.

Definition 6.7. We denote by $\mathcal{F}_{N}$ the set of all elements $\phi \in \mathcal{M}_{N}$ such that $z \mapsto \phi(z)$ is Borel measurable and bounded on $\sigma(\Theta(N)) \backslash V_{\mathbb{R}}(\mathcal{I})$, and such that for each $w \in \sigma(\Theta(N)) \cap V_{\mathbb{R}}(\mathcal{I})$, which is not isolated in $\sigma(\Theta(N))$,

$$
\begin{aligned}
\phi(z)-\left.\phi(w)\right|_{x=\operatorname{Re} z, y=\operatorname{Im} z}=O\left(\chi_{Q(w)}(z)\right) \\
\quad \text { as } \sigma(\Theta(N)) \backslash V_{\mathbb{R}}(\mathcal{I}) \ni z \rightarrow w .
\end{aligned}
$$

Note that in $(6.1) \phi(w) \in \mathcal{A}(w)$ is a coset $p(x, y)+(P(w) \cdot Q(w))$ from $\mathbb{C}[x, y] /(P(w) \cdot Q(w))$, and $\left.\phi(w)\right|_{x=\operatorname{Re} z, y=\operatorname{Im} z}$ stands for any representative of this coset $\phi(w)$ considered as a function of $z$. In (6.1) it does not matter what representative we take since $q=O\left(\chi_{Q(w)}\right)$ as $z \rightarrow w$ for any $q \in Q(w)$, and hence, for any $q \in(P(w) \cdot Q(w))$.

Remark 6.8. Assume that our zero-dimensional ideal $\mathcal{I}$ is generated by two definitizing polynomials $p_{1} \in \mathbb{R}[x], p_{2} \in \mathbb{R}[y]$ as in Example 5.5. For $w \in$ $V_{\mathbb{R}}(\mathcal{I})$, i.e. $(\operatorname{Re} w, \operatorname{Im} w) \in V(\mathcal{I})$, we conclude from (5.4) in Example 5.5 that

$$
\chi_{Q(w)}(z):=\max \left(\left|(\operatorname{Re} z-\operatorname{Re} w)^{\mathfrak{d}_{1}(\operatorname{Re} w)}\right|,\left|(\operatorname{Im} z-\operatorname{Im} w)^{\mathfrak{d}_{2}(\operatorname{Im} w)}\right|\right) .
$$

Therefore, in this case the function class $\mathcal{F}_{N}$ here coincides exactly with the function class $\mathcal{F}_{N}$ introduced in Definition 4.11, [4].

Example 6.9. For $(\xi, \eta) \in V(\mathcal{I}) \backslash \mathbb{R}^{2}$ and $a \in \mathcal{B}((\xi, \eta))$ the function $a \delta_{(\xi, \eta)} \in$ $\mathcal{M}_{N}$, which assumes the value $a$ at $(\xi, \eta)$ and the value zero on the rest of $\left(\sigma(\Theta(N)) \cup V_{\mathbb{R}}(\mathcal{I})\right) \dot{\cup}\left(V(\mathcal{I}) \backslash \mathbb{R}^{2}\right)$, trivially belongs to $\mathcal{F}_{N}$.

Correspondingly, $a \delta_{w} \in \mathcal{F}_{N}$ for $a \in \mathcal{A}(w)$ with a $w \in V_{\mathbb{R}}(\mathcal{I})$, which is an isolated point of $\sigma(\Theta(N)) \cup V_{\mathbb{R}}(\mathcal{I})$.

Remark 6.10. Let $h$ be defined on an open subset $D$ of $\mathbb{R}^{2}$ with values in $\mathbb{C}$. Moreover, assume that for given $m, n \in \mathbb{N}$ the function $h$ is $m+n-1$ times continuously differentiable. Finally, fix $w \in D$.

The well-known Taylor Approximation Theorem from multidimensional calculus then yields

$h(z)=\sum_{j=0}^{m+n-2} \sum_{\substack{k, l \in \mathbb{N}_{0} \\ k+l=j}} \frac{1}{k ! l !} \frac{\partial^{j} h}{\partial x^{k} \partial y^{l}}(w) \operatorname{Re}(z-w)^{k} \operatorname{Im}(z-w)^{l}+O\left(|z-w|^{m+n-1}\right)$

as $z \rightarrow w$. Since

$$
\begin{aligned}
|z-w|^{m+n-1} & \leq 2^{m+n-1} \max \left(|\operatorname{Re}(z-w)|^{m+n-1},|\operatorname{Im}(z-w)|^{m+n-1}\right) \\
& =O\left(\max \left(|\operatorname{Re}(z-w)|^{m},|\operatorname{Im}(z-w)|^{n}\right)\right),
\end{aligned}
$$

and since $\operatorname{Re}(z-w)^{k} \operatorname{Im}(z-w)^{l}=O\left(\max \left(|\operatorname{Re}(z-w)|^{m},|\operatorname{Im}(z-w)|^{n}\right)\right)$ for $k \geq m$ or $l \geq n$, we also have

$$
\begin{aligned}
h(z)= & \sum_{k=0}^{m-1} \sum_{l=0}^{n-1} \frac{1}{k ! l !} \frac{\partial^{k+l} h}{\partial x^{k} \partial y^{l}}(w) \operatorname{Re}(z-w)^{k} \operatorname{Im}(z-w)^{l} \\
& +O\left(\max \left(|\operatorname{Re}(z-w)|^{m},|\operatorname{Im}(z-w)|^{n}\right)\right) .
\end{aligned}
$$


Lemma 6.11. Let $f: \operatorname{dom} f\left(\subseteq \mathbb{C}^{2}\right) \rightarrow \mathbb{C}$ be a function with the properties mentioned in Definition 6.2. Then $f_{N}$ belongs to $\mathcal{F}_{N}$.

Proof. For a $w \in \sigma(\Theta(N)) \cap V_{\mathbb{R}}(\mathcal{I})$, which is not isolated in $\sigma(\Theta(N))$, and $z \in \sigma(\Theta(N)) \backslash V_{\mathbb{R}}(\mathcal{I})$ sufficiently near at $w$ by Remark 6.10 the expression

$$
\begin{aligned}
& f_{N}(z)-\left.f_{N}(w)\right|_{x=\operatorname{Re}} z, y=\operatorname{Im} z \\
& =f(\operatorname{Re} z, \operatorname{Im} z)-\sum_{(k, l) \in J(w)} \frac{1}{k ! l !} \frac{\partial^{k+l} f}{\partial x^{k} \partial y^{l}}(\operatorname{Re} w, \operatorname{Im} w) \\
& \cdot(\operatorname{Re} z-\operatorname{Re} w)^{k}(\operatorname{Im} z-\operatorname{Im} w)^{l}
\end{aligned}
$$

is a $O\left(\max \left(|\operatorname{Re}(z-w)|^{d_{x}(w)},|\operatorname{Im}(z-w)|^{d_{y}(w)}\right)\right)$, and therefore a $O\left(\chi_{Q(w)}(z)\right)$ as $z \rightarrow w$. Consequently $f_{N} \in \mathcal{F}_{N}$.

Lemma 6.12. If $\phi \in \mathcal{F}_{N}$ is such that $\phi(z)$ is invertible in $\mathbb{C}, \mathcal{A}(z), \mathcal{B}(z)$, respectively, for all $z \in\left(\sigma(\Theta(N)) \cup V_{\mathbb{R}}(\mathcal{I})\right) \dot{\cup}\left(V(\mathcal{I}) \backslash \mathbb{R}^{2}\right)$ and such that $0 \in \mathbb{C}$ does not belong to the closure of $\phi\left(\sigma(\Theta(N)) \backslash V_{\mathbb{R}}(\mathcal{I})\right)$, then $\phi^{-1}: z \mapsto \phi(z)^{-1}$ also belongs to $\mathcal{F}_{N}$.

Proof. By the first assumption $\phi^{-1}$ is a well-defined object belonging to $\mathcal{M}_{N}$. Clearly, with $\phi$ also $z \mapsto \phi(z)^{-1}=\frac{1}{\phi(z)}$ is measurable on $\sigma(\Theta(N)) \backslash V_{\mathbb{R}}(\mathcal{I})$. By the second assumption of the present lemma $z \mapsto \phi(z)^{-1}=\frac{1}{\phi(z)}$ is bounded on this set.

It remains to verify $(6.1)$ for $\phi^{-1}$ at each $w \in \sigma(\Theta(N)) \cap V_{\mathbb{R}}(\mathcal{I})$, which is not isolated in $\sigma(\Theta(N)$. To do so, first note that due to $\phi(w)$ 's invertibility for $z \in \sigma(\Theta(N)) \backslash V_{\mathbb{R}}(\mathcal{I})$ sufficiently near at $w$ we have $\left.\phi(w)\right|_{x=\operatorname{Re} z, y=\operatorname{Im} z}=$ $p(z) \neq 0$, where $p(x, y)$ is a representative of $\phi(w)$. Now calculate

$$
\begin{aligned}
& \phi^{-1}(z)-\left.\phi(w)^{-1}\right|_{x=\operatorname{Re} z, y=\operatorname{Im} z} \\
& =\frac{1}{\phi(z)}-\frac{1}{\left.\phi(w)\right|_{x=\operatorname{Re}} z, y=\operatorname{Im} z} \\
& \quad+\frac{1}{\left.\phi(w)\right|_{x=\operatorname{Re} z, y=\operatorname{Im} z}}-\left.\phi(w)^{-1}\right|_{x=\operatorname{Re} z, y=\operatorname{Im} z}
\end{aligned}
$$

The expression in (6.3) can be written as

$$
-\frac{1}{\left.\phi(z) \cdot \phi(w)\right|_{x=\operatorname{Re} z, y=\operatorname{Im} z}} \cdot\left(\phi(z)-\left.\phi(w)\right|_{x=\operatorname{Re} z, y=\operatorname{Im} z}\right) .
$$

Here $\frac{1}{\phi(z)}$ is bounded by assumption. The assumed invertibility of $\phi(w)$ implies $\left.\phi(w)\right|_{x=\operatorname{Re} w, y=\operatorname{Im} w} \neq 0$. Hence, $\frac{1}{\left.\phi(w)\right|_{x=\operatorname{Re} z, y=\operatorname{Im} z}}$ is bounded for $z$ in a certain neighbourhood of $w$. From $\phi \in \mathcal{F}_{N}$ we then conclude that (6.3) is a $O\left(\chi_{Q(w)}(z)\right)$ as $z \rightarrow w$.

The expression in (6.4) can be rewritten as

$$
-\frac{1}{\left.\phi(w)\right|_{x=\operatorname{Re} z, y=\operatorname{Im} z}} \cdot\left(\left.\left.\phi(w)\right|_{x=\operatorname{Re} z, y=\operatorname{Im} z} \cdot \phi(w)^{-1}\right|_{x=\operatorname{Re} z, y=\operatorname{Im} z}-1\right) .
$$

The product in the brackets is a representative of $\phi(w) \cdot \phi(w)^{-1}=1+(P(w)$. $Q(w)) \in \mathcal{A}(w)$. Hence, $(6.4)$ equals to $\frac{1}{\left.\phi(w)\right|_{x=\operatorname{Re} z, y=\operatorname{Im} z}} q(\operatorname{Re} z, \operatorname{Im} z)$ for a $q \in$ 
$(P(w) \cdot Q(w))$, and is therefore a $O\left(\chi_{Q(w)}(z)\right)$ as $z \rightarrow w$. Altogether (6.2) is a $O\left(\chi_{Q(w)}(z)\right)$ as $z \rightarrow w$. Thus, $\phi^{-1} \in \mathcal{F}_{N}$.

\section{Functional Calculus for Zero-Dimensional $\mathcal{I}$}

For the following recall from Remark 6.4 that for $p \in \mathbb{C}[x, y]$ the function $p_{N} \in \mathcal{F}_{N}$ is defined in Definition 6.2.

Lemma 7.1. For each $\phi \in \mathcal{F}_{N}$ there exists $p \in \mathbb{C}[x, y]$ and complex valued $f_{1}, \ldots, f_{m} \in \mathfrak{B}\left(\sigma(\Theta(N)) \cup V_{\mathbb{R}}(\mathcal{I})\right)$ with $f_{j}(z)=0$ for $z \in V_{\mathbb{R}}(\mathcal{I})$ such that

$$
\phi(z)=p_{N}(z)+\sum_{j} f_{j}(z)\left(p_{j}\right)_{N}(z)
$$

for all $z \in \sigma(\Theta(N)) \cup V_{\mathbb{R}}(\mathcal{I})$, and that $\phi((\xi, \eta))=p_{N}((\xi, \eta))$ for all $(\xi, \eta) \in$ $V(\mathcal{I}) \backslash \mathbb{R}^{2}$.

Proof. We apply Proposition 5.4 to $W=V_{\mathbb{R}}(\mathcal{I})$. The fact, that $\psi$ is an isomorphism, then yields the existence of a polynomial $p \in \mathbb{C}[x, y]$ such that $p+(P(w) \cdot Q(w))=\phi(w)$ for all $w \in V_{\mathbb{R}}(\mathcal{I})$ and such that $p+Q((\xi, \eta))=$ $\phi((\xi, \eta))$ for all $(\xi, \eta) \in V(\mathcal{I}) \backslash \mathbb{R}^{2}$.

By Remark 6.4 we have $\phi(w)=p+(P(w) \cdot Q(w))=p_{N}(w) \in \mathcal{A}(w)$ for $w \in V_{\mathbb{R}}(\mathcal{I})$. For $(\xi, \eta) \in V(\mathcal{I}) \backslash \mathbb{R}^{2}$ we have $\phi((\xi, \eta))=p+Q((\xi, \eta))=$ $p_{N}((\xi, \eta)) \in \mathcal{B}((\xi, \eta))$.

For $j=1, \ldots, m$ we set $f_{j}(z):=\frac{\phi(z)-p(z)}{\sum_{k} p_{k}(z)}$ if $z \in \sigma(\Theta(N)) \backslash V_{\mathbb{R}}(\mathcal{I})$ (see Lemma 3.5), and $f_{j}(z)=0$ if $z \in V_{\mathbb{R}}(\mathcal{I})$. On $\sigma(\Theta(N)) \cup V_{\mathbb{R}}(\mathcal{I})$ we then have

$$
\phi(z)=p_{N}(z)+\sum_{j} f_{j}(z)\left(p_{j}\right)_{N}(z) .
$$

It remains to verify that the functions $f_{j}$ are measurable and bounded on $\sigma(\Theta(N)) \backslash V_{\mathbb{R}}(\mathcal{I})$. The measurability easily follows from the definition of $f_{j}$ and the measurability of $\phi$ on this set. Since there are only finitely many points in $V_{\mathbb{R}}(\mathcal{I})$, the measurability of $f_{j}$ on $\sigma(\Theta(N)) \cup V_{\mathbb{R}}(\mathcal{I})$ follows.

Concerning boundedness, note that by Lemma $6.11 \phi-p_{N}$ belongs to $\mathcal{F}_{N}$. Since any representative $\left.\left(\phi-p_{N}\right)(w)\right|_{x=\operatorname{Re} z, y=\operatorname{Im} z}$ of $\left(\phi-p_{N}\right)(w) \in \mathcal{A}(w)$ belongs to $P(w) \cdot Q(w) \subseteq Q(w)$, we have $\left(\phi-p_{N}\right)(z)=O\left(\chi_{Q(w)}(z)\right)$ as $z \rightarrow w$ for any $w \in \sigma(\Theta(N)) \cap V_{\mathbb{R}}(\mathcal{I})$ which is not isolated on $\sigma(\Theta(N))$. By Remark 6.6 and Lemma 3.5 we have $\chi_{Q(w)}(z)=O\left(\sum_{k} p_{k}(z)\right)$ as $z \rightarrow w$ for $z \in \sigma(\Theta(N)) \backslash V_{\mathbb{R}}(\mathcal{I})$. Therefore,

$$
f_{j}(z)=\frac{\phi(z)-p(z)}{\sum_{k} p_{k}(z)}=O(1) \quad \text { as } \quad z \rightarrow w
$$

for $z \in \sigma(\Theta(N)) \backslash V_{\mathbb{R}}(\mathcal{I})$.

Definition 7.2. Let $\Delta$ be the set of all pairs $\left(\phi ;\left(p,\left.f_{1}\right|_{\sigma(\Theta(N))}, \ldots,\left.f_{m}\right|_{\sigma(\Theta(N))}\right)\right)$ such that all assertions from Lemma 7.1 hold true for $\phi$ and $\left(p, f_{1}, \ldots, f_{m}\right) . \diamond$ 
Remark 7.3. It is straight forward to check that $\Delta$ is a linear subspace of $\mathcal{F}_{N} \times(\mathbb{C}[x, y] \times \mathfrak{B}(\sigma(\Theta(N))) \times \cdots \times \mathfrak{B}(\sigma(\Theta(N))))$, i.e. $\Delta$ is a linear relations. Moreover, it is easy to check that with $\left(\phi ;\left(p,\left.f_{1}\right|_{\sigma(\Theta(N))}, \ldots,\left.f_{m}\right|_{\sigma(\Theta(N))}\right)\right)$ also $\left(\phi^{\#} ;\left(p^{\#}, \overline{\left.f_{1}\right|_{\sigma(\Theta(N))}}, \ldots, \overline{\left.\left.f_{m}\right|_{\sigma(\Theta(N))}\right)}\right)\right.$ belongs to $\Delta$; see Remark 4.5.

$\Delta$ is also compatible with multiplication as will be shown next.

Lemma 7.4. If both, $\left(\phi ;\left(p,\left.f_{1}\right|_{\sigma(\Theta(N))}, \ldots,\left.f_{m}\right|_{\sigma(\Theta(N))}\right)\right)$ and $\left(\psi ;\left(q,\left.g_{1}\right|_{\sigma(\Theta(N))}, \ldots,\left.g_{m}\right|_{\sigma(\Theta(N))}\right)\right)$, belong to $\Delta$, then also the pair $\left(\phi \cdot \psi ;\left(r,\left.h_{1}\right|_{\sigma(\Theta(N))}, \ldots,\left.h_{m}\right|_{\sigma(\Theta(N))}\right)\right)$ belongs to $\Delta$, where (see (4.3))

$$
\begin{aligned}
& \left(r,\left.h_{1}\right|_{\sigma(\Theta(N))}, \ldots,\left.h_{m}\right|_{\sigma(\Theta(N))}\right) \\
& \quad=\left(p,\left.f_{1}\right|_{\sigma(\Theta(N))}, \ldots,\left.f_{m}\right|_{\sigma(\Theta(N))}\right)\left(q,\left.g_{1}\right|_{\sigma(\Theta(N))}, \ldots,\left.g_{m}\right|_{\sigma(\Theta(N))}\right) .
\end{aligned}
$$

Proof. On $\sigma(\Theta(N)) \cup V_{\mathbb{R}}(\mathcal{I})$ we have

$$
\phi(z)=p_{N}(z)+\sum_{j} f_{j}(z)\left(p_{j}\right)_{N}(z) \text { and } \psi(z)=q_{N}(z)+\sum_{j} g_{j}(z)\left(p_{j}\right)_{N}(z) \text {. }
$$

Moreover, $f_{j}(z)=0=g_{j}$ for $z \in V_{\mathbb{R}}(\mathcal{I})$, and

$$
\phi((\xi, \eta))=p_{N}((\xi, \eta)), \psi((\xi, \eta))=q_{N}((\xi, \eta)) \quad \text { for all } \quad(\xi, \eta) \in V(\mathcal{I}) \backslash \mathbb{R}^{2} .
$$

Since $p \mapsto p_{N}$ is compatible with multiplication, $r=p \cdot q$ satisfies $(\phi$. $\psi)((\xi, \eta))=r_{N}((\xi, \eta))$ for all $(\xi, \eta) \in V(\mathcal{I}) \backslash \mathbb{R}^{2}$. Clearly, $h_{j}=p g_{j}+q f_{j}+$ $f_{j} \sum_{k=1}^{m} g_{k} p_{k}$ vanishes on $V_{\mathbb{R}}(\mathcal{I})$. For $z \in \sigma(\Theta(N)) \cup V_{\mathbb{R}}(\mathcal{I})$ we have

$$
\begin{aligned}
\phi(z) \psi(z)=p_{N}(z) q_{N}(z)+\sum_{j}\left(p_{N}(z)\right. & g_{j}(z)+q_{N}(z) f_{j}(z) \\
& \left.+f_{j}(z) \sum_{k} g_{k}(z)\left(p_{k}\right)_{N}(z)\right)\left(p_{j}\right)_{N}(z),
\end{aligned}
$$

which, for $z \in V_{\mathbb{R}}(\mathcal{I})$, coincides with $r_{N}(z)=r_{N}(z)+\sum_{j} h_{j}(z)\left(p_{j}\right)_{N}(z)$. For $z \in \sigma(\Theta(N)) \backslash V_{\mathbb{R}}(\mathcal{I})$ the above equation can be written as

$$
\begin{aligned}
\phi(z) \psi(z) & =r(z)+\sum_{j}\left(p(z) g_{j}(z)+q(z) f_{j}(z)+f_{j}(z) \sum_{k} g_{k}(z) p_{k}(z)\right) p_{j}(z) \\
& =r_{N}(z)+\sum_{j} h_{j}(z)\left(p_{j}\right)_{N}(z) .
\end{aligned}
$$

We are going to determine the multivalued part mul $\Delta$ of $\Delta$.

Lemma 7.5. Let $p \in \mathbb{C}[x, y]$ and $f_{1}, \ldots, f_{m} \in \mathfrak{B}\left(\sigma(\Theta(N)) \cup V_{\mathbb{R}}(\mathcal{I})\right)$ with $f_{j}(z)=0$ for $z \in V_{\mathbb{R}}(\mathcal{I})$ be such that

$$
0=p_{N}(z)+\sum_{j} f_{j}(z)\left(p_{j}\right)_{N}(z)
$$

on $\sigma(\Theta(N)) \cup V_{\mathbb{R}}(\mathcal{I})$ and that $p_{N}((\xi, \eta))=0$ for all $(\xi, \eta) \in V(\mathcal{I}) \backslash \mathbb{R}^{2}$. Then $\left(p,\left.f_{1}\right|_{\sigma(\Theta(N))}, \ldots,\left.f_{m}\right|_{\sigma(\Theta(N))}\right)$ belongs to the ideal $\mathcal{N}$ in $\mathcal{R}$ as defined in Definition 4.4 . 
Proof. Clearly, $p+\sum_{j=1}^{m} f_{j} p_{j}=0$ on $\sigma(\Theta(N)) \backslash V_{\mathbb{R}}(\mathcal{I})$. According to Remark 6.4 we have $p+(P(w) \cdot Q(w))=0 \in \mathcal{A}(w)$ for all $w \in V_{\mathbb{R}}(\mathcal{I})$ and $p+Q((\xi, \eta))=0 \in \mathcal{B}((\xi, \eta))$ for all $(\xi, \eta) \in V(\mathcal{I}) \backslash \mathbb{R}^{2}$. Hence, $p$ belongs to

$$
\bigcap_{(\xi, \eta) \in V(\mathcal{I}) \backslash \mathbb{R}^{2}} Q((\xi, \eta)) \cap \bigcap_{w \in V_{\mathbb{R}}(\mathcal{I})}(P(w) \cdot Q(w)) .
$$

By Proposition 5.4 we therefore have $p=\sum_{j} u_{j} p_{j}$ with $u_{j}(w)=0$ for all $w \in V_{\mathbb{R}}(\mathcal{I})$. We see that $\left(f_{j}+u_{j}\right)(z)=0$ for all $z \in V_{\mathbb{R}}(\mathcal{I}) \cap \sigma(\Theta(N))$. Thus, $\left(p,\left.f_{1}\right|_{\sigma(\Theta(N))}, \ldots,\left.f_{m}\right|_{\sigma(\Theta(N))}\right) \in \mathcal{N}$.

Since by Lemma 4.6 mul $\Delta \subseteq \mathcal{N} \subseteq \operatorname{ker} \Psi$, the composition $\Psi \Delta$ is a well-defined linear mapping from $\mathcal{F}_{N}$ into $B(\mathcal{K})$.

Definition 7.6. For $\phi \in \mathcal{F}_{N}$ we set $\phi(N):=(\Psi \Delta)(\phi)$.

By Theorem 4.9, Lemma 7.4 and Remark 7.3 the following result can be formulated.

Theorem 7.7. $\phi \mapsto \phi(N)$ constitutes a $*$-homomorphism from $\mathcal{F}_{N}$ into $\{N$, $\left.N^{*}\right\}^{\prime \prime} \subseteq B(\mathcal{K})$. It satisfies $p_{N}(N)=p(A, B)$ for all $p \in \mathbb{C}[x, y]$.

Proof. The final assertion is clear because of $\left(p_{N} ;(p, 0, \ldots, 0)\right) \in \Delta$.

\section{Spectral Properties of the Functional Calculus}

For $w \in V_{\mathbb{R}}(\mathcal{I})$ we will need the following notation. By $\pi_{w}: \mathcal{A}(w) \rightarrow \mathcal{B}(w)$ we denote the mapping

$$
\pi_{w}(f+(P(w) \cdot Q(w)))=f+Q(w) .
$$

Lemma 8.1. If $\phi \in \mathcal{F}_{N}$ vanishes everywhere except at a fixed $w \in V_{\mathbb{R}}(\mathcal{I})$ and if $\pi_{w} \phi(w)=0$, then

$$
\phi(N)=\Psi\left(0 ; g_{1}, \ldots, g_{m}\right)
$$

for $g_{1}, \ldots, g_{m} \in \mathfrak{B}(\sigma(\Theta(N)))$ which vanish on $\left(\sigma(\Theta(N)) \cup V_{\mathbb{R}}(\mathcal{I})\right) \backslash\{w\}$.

Proof. Let $p \in \mathbb{C}[x, y]$ and $f_{1}, \ldots, f_{m} \in \mathfrak{B}\left(\sigma(\Theta(N)) \cup V_{\mathbb{R}}(\mathcal{I})\right)$ with $f_{j}(z)=0$ for $z \in V_{\mathbb{R}}(\mathcal{I})$ such that

$$
\phi(z)=p_{N}(z)+\sum_{j} f_{j}(z)\left(p_{j}\right)_{N}(z)
$$

for all $z \in \sigma(\Theta(N)) \cup V_{\mathbb{R}}(\mathcal{I})$, and that $p_{N}((\xi, \eta))=\phi((\xi, \eta))=0$ for all $(\xi, \eta) \in V(\mathcal{I}) \backslash \mathbb{R}^{2}$. The latter fact just means $p \in Q((\xi, \eta))$. From $0=\phi(z)=$ $p_{N}(z)+\sum_{j} f_{j}(z)\left(p_{j}\right)_{N}(z)$ for $z \in V_{\mathbb{R}}(\mathcal{I}) \backslash\{w\}$ we infer $p \in(P(z) \cdot Q(z))$. From $\pi_{w} \phi(w)=0$ we obtain $p \in Q(w)$.

By Proposition 5.4 we have $p=\sum_{j} u_{j} p_{j}$, where $u_{j}(z)=0$ for all $z \in V_{\mathbb{R}}(\mathcal{I}) \backslash\{w\}$. We define $g_{j}$ to be zero on $\left(\sigma(\Theta(N)) \cup V_{\mathbb{R}}(\mathcal{I})\right) \backslash\{w\}$ and set $g_{j}(w)=u_{j}(w)$. The difference

$$
\begin{aligned}
& \left(p ;\left.f_{1}\right|_{\sigma(\Theta(N))}, \ldots,\left.f_{m}\right|_{\sigma(\Theta(N))}\right)-\left(0 ; g_{1}, \ldots, g_{m}\right) \\
& \quad=\left(p ;\left.f_{1}\right|_{\sigma(\Theta(N))}-\delta_{w}(.) u_{1}(w), \ldots,\left.f_{m}\right|_{\sigma(\Theta(N))}-\delta_{w}(.) u_{m}(w)\right)
\end{aligned}
$$


satisfies $p+\sum_{j}\left(f_{j}(z)-\delta_{w}(z) u_{j}(w)\right) p_{j}(z)=\phi(z)=0$ for $z \in \sigma(\Theta(N)) \backslash V_{\mathbb{R}}(\mathcal{I})$ and $f_{j}(z)-\delta_{w}(z) u_{j}(w)+u_{j}(z)=0$ for all $z \in V_{\mathbb{R}}(\mathcal{I}) \cap \sigma(\Theta(N))$. It therefore belongs to the ideal $\mathcal{N}$ of $\mathcal{R}$. Thus,

$$
\phi(N)=\Psi\left(p ;\left.f_{1}\right|_{\sigma(\Theta(N))}, \ldots,\left.f_{m}\right|_{\sigma(\Theta(N))}\right)=\Psi\left(0 ; g_{1}, \ldots, g_{m}\right) .
$$

Corollary 8.2. Assume that the spectral measure $E$ of $\Theta(N)$ satisfies $E\{w\}=$ 0 for a fixed $w \in V_{\mathbb{R}}(\mathcal{I})$, which surely happens if $w \notin \sigma(\Theta(N))$. Then $\phi(N)=$ $\psi(N)$ for all $\phi, \psi$ that coincide on $\left(\left(\sigma(\Theta(N)) \cup V_{\mathbb{R}}(\mathcal{I})\right) \backslash\{w\}\right) \cup\left(V(\mathcal{I}) \backslash \mathbb{R}^{2}\right)$ and that satisfy $\pi_{w} \phi(w)=\pi_{w} \psi(w)$. Here $\pi_{w}: \mathcal{A}(w) \rightarrow \mathcal{B}(w)$ is defined by $\pi_{w}(f+$ $(P(w) \cdot Q(w)))=f+Q(w)$.

Proof. By Lemma 8.1 there exist $g_{1}, \ldots, g_{m} \in \mathfrak{B}(\sigma(\Theta(N)))$, which vanish on $\left(\sigma(\Theta(N)) \cup V_{\mathbb{R}}(\mathcal{I})\right) \backslash\{w\}$, such that

$$
\phi(N)-\psi(N)=\Psi\left(0 ; g_{1}, \ldots, g_{m}\right)=\sum_{k=1}^{m} \Xi_{k}\left(\int_{\sigma\left(\Theta_{k}(N)\right)} g_{k} d E_{k}\right)
$$

According to Lemma 4.1 together with our assumption $E\{w\}=0$, this operator vanishes.

Remark 8.3. For $\zeta \in V(\mathcal{I}) \backslash \mathbb{R}^{2}$ or a $\zeta \in V_{\mathbb{R}}(\mathcal{I})$, which is isolated in $\sigma(\Theta(N)) \cup$ $V_{\mathbb{R}}(\mathcal{I})$, we saw in Example 6.9 that $a \delta_{\zeta} \in \mathcal{F}_{N}$. If $a$ is the unite $e$ in $\mathcal{B}(\zeta)$ or in $\mathcal{A}(\zeta)$, i.e. the coset $1+Q(\zeta)$ for $\zeta \in V(\mathcal{I}) \backslash \mathbb{R}^{2}$ or the coset $1+(P(\zeta) \cdot Q(\zeta))$ for $\zeta \in V_{\mathbb{R}}(\mathcal{I})$, then $\left(e \delta_{\zeta}\right) \cdot\left(e \delta_{\zeta}\right)=\left(e \delta_{\zeta}\right)$ together with the multiplicativity of $\phi \mapsto \phi(N)$ show that $\left(e \delta_{\zeta}\right)(N)$ is a projection. It is a kind of Riesz projection corresponding to $\zeta$.

We set $\xi:=\operatorname{Re} \zeta, \eta:=\operatorname{Im} \zeta$ if $\zeta \in V_{\mathbb{R}}(\mathcal{I})$ and $(\xi, \eta):=\zeta$ if $\zeta \in V(\mathcal{I}) \backslash \mathbb{R}^{2}$. For $\lambda \in \mathbb{C} \backslash\{\xi+i \eta\}$ and for $s(z, w):=z+i w-\lambda$ we then have $s_{N} \cdot\left(e \delta_{\zeta}\right)=$ $\left(s_{N}(\zeta)\right) \delta_{\zeta}$. As $s(\xi, \eta) \neq 0, s_{N}(\zeta)$ does not belong to $P(\zeta) \supseteq Q(\zeta)$. Therefore, it is invertible in $\mathcal{B}(\zeta)$ or in $\mathcal{A}(\zeta)$. For its inverse $b$ we obtain

$$
s_{N} \cdot\left(e \delta_{\zeta}\right) \cdot\left(b \delta_{\zeta}\right)=e \delta_{\zeta} .
$$

From $s_{N}(N)=N-\lambda$ we derive that $\left(\left.N\right|_{\operatorname{ran}\left(e \delta_{\zeta}\right)(N)}-\lambda\right)^{-1}=\left(b \delta_{\zeta}\right)$ $\left.(N)\right|_{\operatorname{ran}\left(e \delta_{\zeta}\right)(N)}$ on $\operatorname{ran}\left(e \delta_{\zeta}\right)(N)$. In particular, $\sigma\left(\left.N\right|_{\operatorname{ran}\left(e \delta_{\zeta}\right)(N)}\right) \subseteq\{\xi+i \eta\}$. $\diamond$

Lemma 8.4. If $\phi \in \mathcal{F}_{N}$ vanishes on $\left(\sigma(\Theta(N)) \cup\left(V_{\mathbb{R}}(\mathcal{I}) \cap \sigma(N)\right)\right) \dot{\cup}\left\{(\alpha, \beta) \in V(\mathcal{I}) \backslash \mathbb{R}^{2}: \alpha+i \beta, \bar{\alpha}+i \bar{\beta} \in \sigma(N)\right\}$, then $\phi(N)=0$.

Proof. Since any $w \in V_{\mathbb{R}}(\mathcal{I}) \backslash \sigma(N)$ is isolated in $\sigma(\Theta(N)) \cup V_{\mathbb{R}}(\mathcal{I})$, we saw in Remark 8.3 that for

$$
\zeta \in \underbrace{\left(V_{\mathbb{R}}(\mathcal{I}) \backslash \sigma(N)\right)}_{=: Z_{1}} \dot{ن} \underbrace{\left\{(\alpha, \beta) \in V(\mathcal{I}) \backslash \mathbb{R}^{2}: \alpha+i \beta \in \rho(N)\right\}}_{=: Z_{2}}
$$

the expression $\left(e \delta_{\zeta}\right)(N)$ is a bounded projection commuting with $N$. Hence, $\left(e \delta_{\zeta}\right)(N)$ also commutes with $(N-(\xi+i \eta))^{-1}$, where $\xi:=\operatorname{Re} \zeta, \eta:=\operatorname{Im} \zeta$ if $\zeta \in Z_{1}$ and $(\xi, \eta):=\zeta$ if $\zeta \in Z_{2}$. 
Consequently, $\left.N\right|_{\operatorname{ran}\left(e \delta_{\zeta}\right)(N)}-(\xi+i \eta)$ is invertible on $\operatorname{ran}\left(e \delta_{\zeta}\right)(N)$, i.e. $\xi+i \eta \notin \sigma\left(\left.N\right|_{\operatorname{ran}\left(e \delta_{\zeta}\right)(N)}\right)$. In Remark 8.3 we saw $\sigma\left(\left.N\right|_{\operatorname{ran}\left(e \delta_{\zeta}\right)(N)}\right) \subseteq\{\xi+i \eta\}$. Hence, $\sigma\left(\left.N\right|_{\operatorname{ran}\left(e \delta_{\zeta}\right)(N)}\right)=\emptyset$, which is impossible for $\operatorname{ran}\left(e \delta_{\zeta}\right)(N) \neq\{0\}$. Thus, $\left(e \delta_{\zeta}\right)(N)=0$.

For $(\xi, \eta) \in Z_{3}:=\left\{(\alpha, \beta) \in V(\mathcal{I}) \backslash \mathbb{R}^{2}: \bar{\alpha}+i \bar{\beta} \in \rho(N)\right\}$ one has $(\bar{\xi}, \bar{\eta}) \in$ $Z_{2}$. Hence,

$$
0=\left(e \delta_{(\bar{\xi}, \bar{\eta})}\right)(N)^{*}=\left(e^{\#} \delta_{(\xi, \eta)}\right)(N)=\left(e \delta_{(\xi, \eta)}\right)(N) .
$$

Since, by our assumption, $\phi$ is supported on $Z_{1} \cup Z_{2} \cup Z_{3}$, we obtain

$$
\phi(N)=\left(\sum_{\zeta \in Z_{1} \cup Z_{2} \cup Z_{3}} \phi(\zeta) \delta_{\zeta}\right)(N)=\sum_{\zeta \in Z_{1} \cup Z_{2} \cup Z_{3}} \phi(\zeta)\left(e \delta_{\zeta}\right)(N)=0 .
$$

As a consequence of Lemma 8.4 for $\phi \in \mathcal{F}_{N}$ the operator $\phi(N)$ only depends on $\phi$ 's values on

$$
\begin{aligned}
\left(\sigma ( \Theta ( N ) ) \cup \left(V_{\mathbb{R}}(\mathcal{I}) \cap\right.\right. & \sigma(N))) \\
& \dot{U}\left\{(\alpha, \beta) \in V(\mathcal{I}) \backslash \mathbb{R}^{2}: \alpha+i \beta, \bar{\alpha}+i \bar{\beta} \in \sigma(N)\right\} .
\end{aligned}
$$

Thus, we can, and will from now on, re-define the function class $\mathcal{F}_{N}$ for our functional calculus so that the elements $\phi$ of $\mathcal{F}_{N}$ are functions on this set with values in $\mathbb{C}, \mathcal{A}(z)$ or $\mathcal{B}(z)$, such that $z \mapsto \phi(z)$ is measurable and bounded on $\sigma(\Theta(N)) \backslash V_{\mathbb{R}}(\mathcal{I})$ and such that (6.1) holds true for every $w \in \sigma(\Theta(N)) \cap V_{\mathbb{R}}(\mathcal{I})$ which is not isolated in $\sigma(\Theta(N))$.

Lemma 8.5. If $\phi \in \mathcal{F}_{N}$ is such that $\phi(z)$ is invertible in $\mathbb{C}, \mathcal{A}(z)$ or $\mathcal{B}(z)$, respectively, for all $z$ in (8.1), and such that 0 does not belong to the closure of $\phi\left(\sigma(\Theta(N)) \backslash V_{\mathbb{R}}(\mathcal{I})\right)$, then $\phi(N)$ is a boundedly invertible operator on $\mathcal{K}$ with $\phi^{-1}(N)$ as its inverse.

Proof. We think of $\phi$ as a function on $\left(\sigma(\Theta(N)) \cup V_{\mathbb{R}}(\mathcal{I})\right) \dot{\cup}\left(V(\mathcal{I}) \backslash \mathbb{R}^{2}\right)$ by setting $\phi(z)=e$ for all $z$ not belonging to (8.1). Then all assumptions of Lemma 6.12 are satisfied. Hence $\phi^{-1} \in \mathcal{F}_{N}$, and we conclude from Theorem 7.7 and Remark 6.4 that

$$
\phi^{-1}(N) \phi(N)=\phi(N) \phi^{-1}(N)=\left(\phi \cdot \phi^{-1}\right)(N)=\mathbb{1}_{N}(N)=I_{\mathcal{K}} .
$$

Corollary 8.6. $\sigma(N)$ equals to

$$
\begin{aligned}
& \sigma(\Theta(N)) \cup\left(V_{\mathbb{R}}(\mathcal{I}) \cap \sigma(N)\right) \\
& \cup\left\{\alpha+i \beta:(\alpha, \beta) \in V(\mathcal{I}) \backslash \mathbb{R}^{2}, \alpha+i \beta, \bar{\alpha}+i \bar{\beta} \in \sigma(N)\right\} .
\end{aligned}
$$

In particular, $\sigma(N) \backslash \sigma(\Theta(N))$ is finite.

Proof. Since $\Theta$ is a homomorphism, we have $\sigma(\Theta(N)) \subseteq \sigma(N)$. Hence, (8.2) is contained in $\sigma(N)$. For the converse, consider the polynomial $s(z, w)=$ $z+i w-\lambda$ for a $\lambda$ not belonging to (8.2). We conclude that for any

$$
\zeta \in\left(V_{\mathbb{R}}(\mathcal{I}) \cap \sigma(N)\right) \cup\left\{(\alpha, \beta) \in V(\mathcal{I}) \backslash \mathbb{R}^{2}: \alpha+i \beta, \bar{\alpha}+i \bar{\beta} \in \sigma(N)\right\}
$$


the polynomial $s$ does not belong to $P(\zeta) \supseteq Q(\zeta)$. Hence, $s_{N}(\zeta)$ is invertible $\mathcal{A}(\zeta)$ or $\mathcal{B}(\zeta)$. Clearly, $s_{N}(\zeta) \neq 0$ for $\zeta \in \sigma(\Theta(N)) \backslash V_{\mathbb{R}}(\mathcal{I})$. Finally, 0 does not belong to the closure of

$$
s_{N}\left(\sigma(\Theta(N)) \backslash V_{\mathbb{R}}(\mathcal{I})\right)=s\left(\sigma(\Theta(N)) \backslash V_{\mathbb{R}}(\mathcal{I})\right) \subseteq \sigma(\Theta(N))-\lambda .
$$

Applying Lemma 8.5, we see that $s_{N}(N)=(N-\lambda)$ is invertible.

Remark 8.7. We set $K_{r}:=V_{\mathbb{R}}(\mathcal{I}) \cap \sigma(N)$,

$$
Z:=\left\{(\alpha, \beta) \in V(\mathcal{I}) \backslash \mathbb{R}^{2}: \alpha+i \beta, \bar{\alpha}+i \bar{\beta} \in \sigma(N)\right\},
$$

and $K_{i}:=\{\alpha+i \beta:(\alpha, \beta) \in Z\}$. Using Corollary 8.6 we could re-define once more the functions $\phi \in \mathcal{F}_{N}$ as functions $\phi$ on $\sigma(N)$ such that

1. $\phi$ is complex valued, bounded and measurable on $\sigma(N) \backslash\left(K_{r} \cup K_{i}\right)$,

2. $\phi(\zeta) \in \mathcal{A}(\zeta)$ for $\zeta \in K_{r} \backslash K_{i}$,

3. $\phi(\zeta) \in X_{(\alpha, \beta) \in Z, \alpha+i \beta=\zeta} \mathcal{A}(\zeta)$ for $\zeta \in K_{i} \backslash K_{r}$,

4. $\phi(\zeta) \in \mathcal{A}(\zeta) \times X_{(\alpha, \beta) \in Z, \alpha+i \beta=\zeta} \mathcal{A}(\zeta)$ for $\zeta \in K_{r} \cap K_{i}$;

5. for a $w \in K_{r}$, which is not isolated in $\sigma(N)$, we have

$$
\phi(z)-p(\operatorname{Re} z, \operatorname{Im} z)=O\left(\chi_{Q(w)}(z)\right) \quad \text { as } \quad \sigma(N) \backslash\left(K_{r} \cup K_{i}\right) \ni z \rightarrow w,
$$

where $p$ is a representative of $\phi(w)$ for $w \in K_{r} \backslash K_{i}$ and $p$ is a representative of the first entry of $\phi(w)$ for $w \in K_{r} \cap K_{i}$.

\section{Special Cases of Definitizable Operators}

Unitary and selfadjoint operators are special cases of normal operators on Hilbert spaces as well as on Krein spaces. We will show how some well-known facts on definitizable selfadjoint or unitary operators on a Krein space $\mathcal{K}$ can easily be obtain from the previously obtained results.

\subsection{Selfadjoint Definitizable Operators}

An operator $N \in B(\mathcal{K})$ is by definition selfadjoint if $N=N^{+}$. Obviously, $N \in B(\mathcal{K})$ is selfadjoint if and only if $N$ is normal and satisfies $p(A, B)=0$, where $A=\frac{N+N^{+}}{2}, B=\frac{N-N^{+}}{2 i}$ and $p(x, y)=y$.

Therefore, according to Definition 3.1 any selfadjoint operator on a Krein space is definitizable normal, and the ideal $\mathcal{I}$ generated by all real definitizing polynomials contains $p(x, y)=y$. Since the ideal generated by $p(x, y)=y$ is not zero-dimensional, the zero-dimensionality of $\mathcal{I}$ implies the existence of at least one real definitizing polynomial of the form

$$
y \cdot s(x, y)+t(x) \quad \text { with } s \in \mathbb{C}[x, y], t \in \mathbb{C}[x] \backslash\{0\} .
$$

Proposition 9.1. The ideal $\mathcal{I}$ is zero-dimensional if and only if there exists a $t \in \mathbb{R}[x] \backslash\{0\}$ such that $[t(A) u, u] \geq 0, u \in \mathcal{K}$, i.e. $N=A$ is definitizable in the classical sense; see [5].

Proof. Any $r \in \mathbb{C}[x, y]$ can we written as $r(x, y)=y \cdot s_{r}(x, y)+t_{r}(x)$ with unique $s_{r} \in \mathbb{C}[x, y], t_{r} \in \mathbb{C}[x]$. Hence, $r \in \mathcal{I}$ if and only if $t_{r} \in \mathcal{I}$. The set of $\mathcal{I}_{x}:=\left\{t_{r}: r \in \mathcal{I}\right\}$ forms an ideal in $\mathbb{C}[x]$. If $\mathcal{I}_{x}$ is the zero ideal, then $\mathcal{I}=y \cdot \mathbb{C}[x, y]$ is not zero-dimensional. 
If $\mathcal{I}_{x} \neq\{0\}$, then, applying the polynomial division, we see that dim $\mathbb{C}[x] / \mathcal{I}_{x}<\infty$. This also implies the zero-dimensionality of $\mathcal{I}$. If $r(x, y)$ is a real definitizing polynomial as in (9.1), then

$$
[t(A) u, u]=[r(A, B) u, u] \geq 0, u \in \mathcal{K},
$$

i.e. $t(x)$ is a definitizing polynomial. Finally, $r$ shares the property to be real with $t$.

Assume that $N \in B(\mathcal{K})$ is selfadjoint and that the ideal $\mathcal{I}$ generated by all real definitizing polynomials is zero-dimensional. Consequently, we can apply the functional calculus developed in Section 7 . Since $p(x, y)=y$ belongs to $\mathcal{I}$, we conclude that

$$
a=\left(a_{x}, a_{y}\right) \in V(\mathcal{I}) \quad \text { implies } \quad a_{y}=p(a)=0 .
$$

Hence, the elements of $V_{\mathbb{R}}(\mathcal{I})$ are contained in $\mathbb{R}$, and $(\xi, \eta) \in V(\mathcal{I}) \backslash \mathbb{R}^{2}$ yields $\eta=0$. Moreover, with $N$ also $\Theta(N)$ is selfadjoint in the Hilbert space $\mathcal{H}$; see Proposition 3.3 and (2.1). In particular, $\sigma(\Theta(N)) \subseteq \mathbb{R}$. From Corollary 8.6 we derive that $\sigma(N)$ is contained in $\mathbb{R}$ up to finitely many points which are located in $\mathbb{C} \backslash \mathbb{R}$ symmetric with respect to $\mathbb{R}$.

\subsection{Unitary Definitizable Operators}

An operator $N \in B(\mathcal{K})$ is by definition unitary if $N^{+} N=N N^{+}=I_{\mathcal{K}}$. Obviously, $N \in B(\mathcal{K})$ is unitary if and only if $N$ is normal and satisfies $p(A, B)=0$, where $A=\frac{N+N^{+}}{2}, B=\frac{N-N^{+}}{2 i}$ and

$$
p(x, y)=(x+i y)(x-i y)-1=x^{2}+y^{2}-1 .
$$

Therefore, according to Definition 3.1 any unitary operator on a Krein space is definitizable normal, and the ideal $\mathcal{I}$ generated by all real definitizing polynomials always contains $p(x, y)$. Since the ideal generated by $p$ is not zero-dimensional, the zero-dimensionality of $\mathcal{I}$ implies the existence a definitizing polynomial different from $p$.

Remark 9.2. If, for example, there exists a polynomial $a \in \mathbb{C}[z] \backslash\{0\}$ such that $[a(N) u, u] \geq 0, u \in \mathcal{K}$, then the ideal $\mathcal{J}$ generated by $a$ (as a polynomial in $\mathbb{C}[z, w])$ and $b(z, w)=z w-1$ in $\mathbb{C}[z, w]$ is zero-dimensional. Indeed, it is easy to see that the set $V(\mathcal{J})$ of common zeros of $a$ and $b$ is finite, which by [3], page 39, implies zero-dimensionality. Since $c(z, w) \mapsto c(x+i y, x-i y)$ constitutes an isomorphism from $\mathbb{C}[z, w]$ onto $\mathbb{C}[x, y]$, also the ideal generated by $a(x+i y)$ and $p(x, y)$ in $\mathbb{C}[x, y]$ is zero-dimensional. Hence, the same is true for $\mathcal{I}$, and we can apply the functional calculus developed Section 7 .

Assume that $N \in B(\mathcal{K})$ is unitary and that the ideal $\mathcal{I}$ generated by all real definitizing polynomials is zero-dimensional. Consequently, we can apply the functional calculus developed in Section 7. From $p \in \mathcal{I}$ we conclude that

$$
a \in V(\mathcal{I}) \text { implies } p(a)=0 .
$$

Hence, the elements of $V_{\mathbb{R}}(\mathcal{I})$ are contained in $\mathbb{T}$, and $(\xi, \eta) \in V(\mathcal{I}) \backslash \mathbb{R}^{2}$ yields

$$
(\xi+i \eta) \overline{(\bar{\xi}+i \bar{\eta})}=\xi^{2}+\eta^{2}=1 .
$$


Moreover, with $N$ also $\Theta(N)$ is unitary in the Hilbert space $\mathcal{H}$; see Proposition 3.3 and (2.1). In particular, $\sigma(\Theta(N)) \subseteq \mathbb{T}$. From Corollary 8.6 we derive that $\sigma(N)$ is contained in $\mathbb{T}$ up to finitely many points which are located in $\mathbb{C} \backslash \mathbb{T}$ symmetric with respect to $\mathbb{T}$.

\section{Transformations of Definitizable Normal Operators}

In this final section we examine, whether basic transformations, such as $\alpha N, N+\beta I_{\mathcal{K}}, N^{-1}$ with $\alpha, \beta \in \mathbb{C}, \alpha \neq 0$, of definitizable normal operators $N$ are again definitizable, and how the corresponding ideals $\mathcal{I}$ behave.

For $\beta \in \mathbb{C}$ it is easy to see that $p(x, y)$ is a real definitizing polynomial for $N$ if and only if the polynomial $p(x-\operatorname{Re} \beta, y-\operatorname{Im} \beta)$ in $\mathbb{C}[x, y]$ is real definitizing for $N+\beta I_{\mathcal{K}}$. Since $r(x, y) \mapsto r(x-\operatorname{Re} \beta, y-\operatorname{Im} \beta)$ is a ring automorphism on $\mathbb{C}[x, y]$, the respective ideals $\mathcal{I}$, corresponding to $N$ and $N+\beta I_{\mathcal{K}}$, are zero-dimensional, or not, at the same time.

Similarly, $p(x, y)$ is a real definitizing polynomial for $N$ if and only if the polynomial $p(x \operatorname{Re} 1 / \alpha-y \operatorname{Im} 1 / \alpha, x \operatorname{Im} 1 / \alpha+y \operatorname{Re} 1 / \alpha)$ in $\mathbb{C}[x, y]$ is real definitizing for $\alpha N$. Also $r(x, y) \mapsto r(x \operatorname{Re} 1 / \alpha-y \operatorname{Im} 1 / \alpha, x \operatorname{Im} 1 / \alpha+y \operatorname{Re} 1 / \alpha)$ is a ring automorphism on $\mathbb{C}[x, y]$. Hence, the ideal $\mathcal{I}$ corresponding to $N$ is zero-dimensional if and only if the ideal $\mathcal{I}$ corresponding to $\alpha N$ is zerodimensional.

For the inverse $N^{-1}$ the situation is more complicated. We formulate two results that we will need. The first assertion is straight forward to verify. We omit its proof.

Lemma 10.1. The mapping $\Phi: p(x, y) \mapsto p\left(\frac{z+w}{2}, \frac{z-w}{2 i}\right)$ from $\mathbb{C}[x, y]$ to $\mathbb{C}[z, w]$ is an isomorphism, where $p$ is real, i.e. $p(\bar{x}, \bar{y})=\overline{p(x, y)}$, if and only if $\overline{\Phi(p)(z, w)}=\Phi(p)(\bar{w}, \bar{z})$.

Obviously, for a normal $N=A+i B$ and $p(x, y) \in \mathbb{C}[x, y]$ we have

$$
p(A, B)=\Phi(p)\left(N, N^{+}\right) .
$$

For a polynomial $q \in \mathbb{C}[z, w] \backslash\{0\}$ let $d(q)$ be the maximum of the $z$ degree of $q$ and the $w$-degree of $q$. Moreover, we set

$$
\varpi(q)(z, w):=(z w)^{d(q)} q\left(\frac{1}{z}, \frac{1}{w}\right) \in \mathbb{C}[z, w] .
$$

Lemma 10.2. If $\mathcal{I}=\left\langle q_{1}, \ldots, q_{m}\right\rangle$ is zero-dimensional with polynomials $q_{1}, \ldots$, $q_{m}$ such that $\overline{q_{j}(z, w)}=q_{j}(\bar{w}, \bar{z})$, then the ideal $\left\langle\varpi\left(q_{1}\right), \ldots, \varpi\left(r_{m}\right)\right\rangle$ is also zero-dimensional.

Proof. Let $(\zeta, \eta) \in V\left(\varpi\left(q_{1}\right), \ldots, \varpi\left(r_{m}\right)\right)$. For $\zeta \neq 0 \neq \eta$ we conclude $q_{j}\left(\frac{1}{\zeta}, \frac{1}{\eta}\right)$ $=0, j=1, \ldots, m$, and in turn $(\zeta, \eta) \in\left\{(z, w) \in(\mathbb{C} \backslash\{0\})^{2}:\left(\frac{1}{z}, \frac{1}{w}\right) \in V(\mathcal{I})\right\}$.

Assume that $\eta=0$ and $\zeta \neq 0$. If $q_{j}(z, w)=\sum_{k, l=0}^{d\left(q_{j}\right)} b_{k, l} z^{k} w^{l}$, then $\overline{q_{j}(z, w)}=q_{j}(\bar{w}, \bar{z})$ yields $b_{k, l}=\bar{b}_{l, k}$, and we have $\varpi\left(q_{j}\right)(z, w)=\sum_{k, l=0}^{d\left(q_{j}\right)}$ 
$b_{d\left(q_{j}\right)-k, d\left(q_{j}\right)-l} z^{k} w^{l}$. According to the choice of $d\left(q_{j}\right)$ and by $b_{k, l}=\bar{b}_{l, k}$ the polynomial

$$
\rho_{j}(z):=\varpi\left(q_{j}\right)(z, 0)=\sum_{k=0}^{d\left(q_{j}\right)} b_{d\left(q_{j}\right)-k, d\left(q_{j}\right)} z^{k}
$$

is non-zero and satisfies $\rho_{j}(\zeta)=0$, i.e. $(\zeta, \eta) \in \rho_{j}^{-1}(\{0\}) \times\{0\}$.

From $\overline{q_{j}(z, w)}=q_{j}(\bar{w}, \bar{z})$ we conclude $\rho_{j}(\bar{w})=\overline{\varpi\left(q_{j}\right)(0, w)}$. Hence, $\zeta=0$ and $\eta \neq 0$ yields $(\zeta, \eta) \in\{0\} \times \overline{\rho_{j}^{-1}(\{0\})}$.

In any case $(\zeta, \eta)$ is contained in

$$
\begin{aligned}
& \{(0,0)\} \cup\left\{(z, w) \in(\mathbb{C} \backslash\{0\})^{2}:\left(\frac{1}{z}, \frac{1}{w}\right) \in V(\mathcal{I})\right\} \\
& \cup\left(\bigcap_{j=1, \ldots, m} \rho_{j}^{-1}(\{0\}) \times\{0\}\right) \cup\left(\bigcap_{j=1, \ldots, m}\{0\} \times \overline{\rho_{j}^{-1}(\{0\})}\right) .
\end{aligned}
$$

Consequently, $V\left(\varpi\left(q_{1}\right), \ldots, \varpi\left(r_{m}\right)\right)$ is finite, and in turn $\left\langle\varpi\left(q_{1}\right), \ldots, \varpi\left(r_{m}\right)\right\rangle$ is zero-dimensional; see [3], page 39 .

Proposition 10.3. Let $N$ be normal and bijective on the Krein space $\mathcal{K}$. If $p(x, y)$ is real definitizing for $N$, then $\Phi^{-1}(\varpi(\Phi(p)))$ is definitizing for $N^{-1}$. Moreover, if the ideal I generated by all real definitizing $p(x, y)$ for $N$ is zerodimensional, then also the ideal generated by all real definitizing polynomials for $N^{-1}$ is zero-dimensional.

Proof. Let $p(x, y)$ be real definitizing for $N$. By Lemma 10.1 we have $\overline{\Phi(p)(z, w)}=\Phi(p)(\bar{w}, \bar{z})$, and in turn $\overline{\varpi(\Phi(p))(z, w)}=\varpi(\Phi(p))(\bar{w}, \bar{z})$. Writing $\Phi(p)(z, w)=\sum_{k, l=0}^{d(\Phi(p))} b_{k, l} z^{k} w^{l}$, we obtain

$$
\varpi(\Phi(p))(z, w)=\sum_{k, l=0}^{d(\Phi(p))} b_{d(\Phi(p))-k, d(\Phi(p))-l} z^{k} w^{l} .
$$

For $u \in \mathcal{K}$ by (10.1) we have

$$
\begin{aligned}
{\left[\Phi^{-1}\right.} & \left.(\varpi(\Phi(p)))\left(\operatorname{Re} N^{-1}, \operatorname{Im} N^{-1}\right) u, u\right] \\
& =\left[\varpi(\Phi(p))\left(N^{-1}, N^{-+}\right) u, u\right] \\
& =\left[\sum_{k, l=0}^{d(\Phi(p))} b_{d(\Phi(p))-k, d(\Phi(p))-l}\left(N^{-1}\right)^{k}\left(N^{-+}\right)^{l} u, u\right] \\
& =\left[\Phi(p)\left(N, N^{+}\right)\left(N^{-1}\right)^{d(\Phi(p))} u,\left(N^{-1}\right)^{d(\Phi(p))} u\right] \\
& =\left[p(A, B)\left(N^{-1}\right)^{d(\Phi(p))} u,\left(N^{-1}\right)^{d(\Phi(p))} u\right] \geq 0 .
\end{aligned}
$$

Hence, $\Phi^{-1}(\varpi(\Phi(p)))$ is real definitizing for $N^{-1}$. Finally, if $\mathcal{I}$ is zerodimensional and generated by real definitizing $p_{1}, \ldots, p_{m}$, then $\Phi(\mathcal{I})=\left\langle\Phi\left(p_{1}\right)\right.$, $\left.\ldots, \Phi\left(p_{m}\right)\right\rangle$ is zero-dimensional in $\mathbb{C}[z, w]$. According to Lemma $10.2\langle\varpi(\Phi$ 
$\left.\left.\left(p_{1}\right)\right), \ldots, \varpi\left(\Phi\left(p_{m}\right)\right)\right\rangle$, and hence also $\left\langle\Phi^{-1}\left(\varpi\left(\Phi\left(p_{1}\right)\right)\right), \ldots, \Phi^{-1}(\varpi(\Phi\right.$ $\left.\left.\left.\left(p_{m}\right)\right)\right)\right\rangle$ is zero-dimensional. Since its generators are real definitizing for $N^{-1}$ also the ideal generated by all real definitizing polynomials for $N^{-1}$ is zerodimensional.

\section{Acknowledgements}

Open access funding provided by TU Wien (TUW).

Open Access. This article is distributed under the terms of the Creative Commons Attribution 4.0 International License (http://creativecommons.org/licenses/ by/4.0/), which permits unrestricted use, distribution, and reproduction in any medium, provided you give appropriate credit to the original author(s) and the source, provide a link to the Creative Commons license, and indicate if changes were made.

\section{References}

[1] Becker, Th., Weispfenning, V., Kredel, H.: Gröbner Bases : A Computational Approach to Commutative Algebra, Graduate Texts in Mathematics. Springer, New York (1993)

[2] Cox, D.A., Little, J., O'Shea, D.: Ideals, Varieties, and Algorithms, Undergraduate Texts in Mathematics. Springer, New York (2007)

[3] Cox, D.A., Little, J., O'Shea, D.: Using Algebraic Geometry Graduate Texts in Mathematics. Springer, New York (1998)

[4] Kaltenbäck, M.: Functional Calculus for definitizable normal linear operators on Krein spaces. Integr. Equ. Oper. Theory 85(2), 221-243 (2016)

[5] Kaltenbäck, M., Pruckner, R.: Functional Calculus for definitizable selfadjoint linear relations on Krein spaces. Integr. Equ. Oper. Theory 83(4), 451-482 (2015)

[6] Langer, H.: Spectral functions of definitizable operators in Krein spaces. Lecture Notes Math. 948, 1-46 (1982)

Michael Kaltenbäck $(\bowtie)$

Wiedner Hauptstr. 8-10

1040 Wien

Austria

e-mail: michael.kaltenbaeck@tuwien.ac.at

Received: March 10, 2016.

Revised: February 11, 2017. 